\title{
CRECIMIENTO, EVAPOTRANSPIRACIÓN Y USO DE NUTRIENTES EN CULTIVO HIDROPÓNICO DE Eryngium foetidum, EN DOS DIFERENTES AMBIENTES Y NIVELES DE NUTRICIÓN
}

\author{
Freddy Soto-Bravo ${ }^{1 / *}$, Gustavo Alonso Rodríguez-Ocampo ${ }^{2}$ \\ Palabras clave: Absorción de nutrientes; concentración de absorción; tasa de crecimiento del cultivo; \\ transpiración del cultivo; culantro coyote. \\ Keywords: Nutrient absorption; absorption concentration; crop growth rate; crop transpiration; wild coriander.
}

Recibido: 02/04/2020

Aceptado: 30/06/2020

RESUMEN

Introducción. La interacción entre genotipo, clima y manejo agronómico determinan el crecimiento del cultivo, y consecuentemente su demanda hídrica y nutricional. Objetivo. Evaluar el efecto del uso de protección y el nivel de nutrición sobre el crecimiento, uso de nutrientes y evapotranspiración del cultivo hidropónico de culantro coyote. Materiales y métodos. El estudio se realizó en 2016, en la Estación Experimental Agrícola Fabio Baudrit Moreno, Alajuela, Costa Rica. Comprendió 2 etapas del cultivo: I) época seca protegido con sarán y II) época lluviosa con plástico. Se aplicaron 4 tratamientos que consistieron en 2 ambientes: un cultivo protegido $(\mathrm{CP})$ y otro sin protección (SP) combinados con 2 niveles de nutrición: alta (NA) y baja concentración de nutrientes (NB). Se utilizó un diseño de 5 bloques al azar con arreglo bifactorial 2 × 2. Se evaluó materia seca (MS), índice de área foliar (IAF) y tasa de crecimiento del cultivo (TCC); así como la evapotranspiración

\footnotetext{
* Autor para correspondencia. Correo electrónico: freddy.sotobravo@ucr.ac.cr

1 Universidad de Costa Rica, Estación Experimental Agrícola Fabio Baudrit Moreno, Costa Rica. (iD) 0000-0003-1959-9597.
}

\begin{abstract}
Growth, evapotranspiration and use of nutrients of the hydroponic crop of Eryngium foetidum, in two different environments and nutrition levels. Introduction. The interaction between genotype, climate and agronomic management practices determines the growth of the crop, and consequently its water and nutritional demand. Objective. The effect of protection and nutrition level on the growth, use of nutrients and evapotranspiration in the hydroponic crop of wild coriander, was evaluated. Materials and methods. A study was conducted in 2016, at the Fabio Baudrit Moreno Agricultural Experimental Station, Alajuela, Costa Rica. It comprised 2 consecutive stages of a growing cycle: I) in dry season protected with saran and II) rainy season covered with plastic. Four treatments were applied that combined 2 environmental conditions: a protected crop (PC) and another without protection (WP); and 2 levels of nutrition: high (HN) and low

\footnotetext{
2 Universidad de Costa Rica, Estación Experimental Agrícola Fabio Baudrit Moreno, Costa Rica. (D) 0000-0001-6033-684X.
} 
del cultivo $\left(\mathrm{ET}_{\mathrm{c}}\right)$ y la extracción, lixiviación y eficiencia de recuperación (ER), tasa de absorción (TA) y concentración de absorción (CA) de nutrientes. Resultados. Bajo protección, mejoró la ER debido a un incremento del crecimiento (MS, IAF, TCC), un aumento en la absorción y una disminución de la lixiviación de nutrientes; además, incrementó la $\mathrm{CA}$ al disminuir la $\mathrm{ET}_{\mathrm{c}}$. Con alto nivel de nutrición, incrementó el crecimiento, la absorción de nutrientes y la CA. No obstante, el elevado incremento en la lixiviación redujo la ER. Conclusión. La mayor producción de biomasa se presentó en los cultivos protegidos y con NA. Sin embargo, con NA se presentó un sensible aumento de la lixiviación que redujo la ER. La protección del cultivo junto al ajuste en la concentración de nutrientes a partir de la CA, permitió integrar el crecimiento con el consumo de agua y nutrientes, mejorando así, la ER sin sacrificar rendimiento.

\section{INTRODUCCIÓN}

Costa Rica es el principal exportador de Eryngium foetidum, en América Central, hacia Estados Unidos y Canadá (Cerdas y Montero 2016), actividad que beneficia cerca de 200 familias de personas agricultoras de las provincias de Limón y Cartago, con un valor anual estimado en \$5,1 millones (Lavagni y Rodríguez 2009).

Las prácticas actuales del sistema de cultivo de culantro coyote, en suelo y a campo abierto basado en el uso intensivo de agroquímicos, ha generado problemas tales como falta de inocuidad del producto, contaminación ambiental y bajos rendimientos que repercuten en baja rentabilidad y colocan en riesgo la estabilidad del mercado de exportación. Además, en general, se utilizan programas de fertilización estándar concentration of nutrients (LN). A randomized block design with a $2 \times 2$ bifactorial arrangement was used. Dry matter (MS), leaf area index (LAI) and crop growth rate (CGR), extraction, leaching, efficiency recovery (ER), absorption rate (AR) and absorption concentration (AC) of nutrients and crop evapotranspiration $\left(\mathrm{ET}_{\mathrm{c}}\right)$, were evaluated. Results. Under protection, improved ER due to increased growth (DM, LAI, CGR) and absorption and decreased leaching; in addition, AC increased by decreasing $\mathrm{ET}_{\mathrm{c}}$. With a high level of nutrition, increased growth, nutrient absorption and AC. However, the high increase in leaching reduced RE. Conclusion. The highest biomass production occurred in protected crops with high nutrition (HN). However, with NA, there was a noticeable increase in leaching that reduced ER. The protection of the crop together with the adjustment of the concentration of nutrients from the $\mathrm{CA}$, allowed to integrate the growth with the consumption of water and nutrients, which improves the ER without sacrificing yield.

sin considerar el requerimiento del cultivo y los aportes del suelo. Ante este panorama, una alternativa es el sistema de cultivo hidropónico, el cual permite mejorar la eficiencia de uso de agua y los fertilizantes, el rendimiento y la calidad del producto (Jara 2016). Es un sistema aislado del suelo, donde cada contenedor viene a ser un lisímetro que facilita la trazabilidad de entradas y salidas de agua y nutrientes (mg. $\mathrm{L}^{-1}$ ), que permite determinar con precisión los requerimientos del cultivo en función del genotipo, el clima y las prácticas de manejo.

Aunque existen suficientes estudios de absorción en cultivos convencionales en suelo a campo abierto, en culantro coyote, únicamente se reporta un estudio realizado por Bertsch (2003), mientras que en hidroponía, específicamente en culantro coyote, no se encontraron estudios 
de absorción de nutrientes. La aplicación de los requerimientos nutricionales determinados en términos de $\mathrm{kg} \cdot \mathrm{ha}^{-1}$ para el cultivo convencional en suelo a campo abierto, al cultivo sin suelo, requiere de un análisis crítico. En fertirrigación convencional en suelo, los requerimientos se aportan en $\mathrm{kg} \cdot \mathrm{ha}^{-1}$ basados en las curvas de absorción, y en su gran mayoría, sin considerar la relación con la evapotranspiración del cultivo $\left(\mathrm{ET}_{\mathrm{c}}\right)$. Por el contrario, en hidroponía, los requerimientos nutricionales del cultivo se aportan como concentración (mg. $\left.\mathrm{L}^{-1}\right)$ de nutrientes $(\mathrm{mg})$ disueltos en el agua que demanda la planta $\left(\mathrm{ET}_{\mathrm{c}}\right.$ : L.m ${ }^{-2}$ ) (Soto y Ramírez 2005), y no en kg.ha ${ }^{-1}$ como sucede en suelo.

Ante la falta de estudios de absorción en hidroponía, una práctica habitual en la producción de cultivos hidropónicos de hojas es aplicar una concentración constante de nutrientes (mg.L $\left.{ }^{-1}\right)$ mediante una solución nutritiva estándar durante todo el ciclo de cultivo, sin considerar los requerimientos del cultivo según patrón de crecimiento en respuesta a las condiciones del clima y las prácticas de manejo agronómico. Esto hace que los aportes de agua y nutrientes en los cultivos hidropónicos varíe ampliamente, lo que reduce su eficiencia de uso.

Para optimizar los aportes de agua y nutrientes a la demanda del cultivo en sus diferentes etapas de crecimiento, un parámetro apropiado es la concentración de absorción de nutrientes (CA: mg. $\left.\mathrm{L}^{-1}\right)$. La CA relaciona la cantidad de nutriente absorbido (mg.m ${ }^{-2}$ ) con la evapotranspiración del cultivo $\left(\mathrm{ET}_{\mathrm{c}}\right.$ : $\mathrm{L} . \mathrm{m}^{-2}$ ) (Sonneveld 2002), según patrón de crecimiento en respuesta al genotipo, condiciones climáticas y prácticas de manejo. Así, la CA (mg.L $\left.\mathrm{L}^{-1}\right)$ viene a representar la auténtica curva de absorción en cultivos hidropónicos, la cual se aplica mediante una solución nutritiva con bajas concentraciones de nutrientes $\left(\mathrm{mg}_{\mathrm{L}} \mathrm{L}^{-1}\right)$ directamente al sustrato en riegos cortos y frecuentes, para asegurar una adecuada disponibilidad y concentración de nutrientes $\left(\mathrm{mg} . \mathrm{L}^{-1}\right)$ en la rizosfera prácticamente en tiempo real.
Además, las diferencias físico-químicas entre un suelo y un sustrato, como medios de cultivo, hacen que el manejo del riego y la nutrición sea diferente. A diferencia del suelo, en hidroponía, el volumen de sustrato disponible por planta es muy pequeño, en el presente estudio fue tan solo 2 L.planta ${ }^{-1}$. En su mayoría, los sustratos utilizados en cultivo sin suelo son inertes, con baja capacidad de retención de agua y de nutrientes, así como una alta capacidad de aireación y drenaje. Dichas características hacen que el sistema de cultivo requiera un mayor nivel de humedad en sustrato dentro de un rango de potencial hídrico de -1 y -10 centibares; mientras que en cultivo en suelo es posible manejar contenidos de humedad a potenciales hídricos por encima de -33 centibares.

La protección del cultivo es una práctica que se ha adoptado recientemente en producción hidropónica en bancales, para reducir los efectos negativos de climas extremos, y para mejorar la eficiencia de uso del agua y los fertilizantes. La protección en época lluviosa reduce el lixiviado de nutrientes y la incidencia de enfermedades por exceso de lluvia (Rojas y Paniagua 2015). En época de verano, amortigua los efectos del exceso de radiación solar, tales como la fotoinhibición que resulta en una disminución de hasta un 50\% en la fotosíntesis (Caseira-Posada 2007) o de la fotooxidación que se manifiesta en quemas de hojas y frutos.

Adicionalmente, el incremento progresivo en el costo de los fertilizantes y la potencial crisis de los recursos hídricos, plantean la necesidad de mejorar la eficiencia del uso del agua y los nutrientes, y así, reducir los costos de producción e incrementar los rendimientos para asegurar la sostenibilidad de los sistemas agrícolas.

El objetivo de este estudio fue evaluar el efecto del uso de la protección y el nivel de nutrición sobre sobre el crecimiento, la evapotranspiración y el uso nutrientes en el cultivo hidropónico de culantro coyote. 


\section{MATERIALES Y MÉTODOS}

El estudio fue realizado entre abril y agosto del 2016, en la Estación Experimental Fabio Baudrit Moreno (EEFBM), ubicada en San José de Alajuela, Costa Rica $\left(10^{\circ} 01^{\prime} \mathrm{N}\right.$ y $84^{\circ} 16^{`} \mathrm{O}, 840 \mathrm{msnm}$ ), con valores promedio anual de temperatura y humedad relativa y precipitación acumulada de $22^{\circ} \mathrm{C}, 78 \%$ y $1940 \mathrm{~mm}$, respectivamente.
Se utilizó un sistema de cultivo hidropónico en sustrato sólido, en contenedores de madera (12 m x $1 \mathrm{~m} \times 0,1 \mathrm{~m}$ ) elevados a $0,8 \mathrm{~m}$ sobre el nivel del suelo e impermeabilizados con plástico negro $(7 \mu \mathrm{m})$. Para evacuar el exceso de agua, se realizó un orificio de drenaje a un $\mathrm{cm}$ del fondo de la cama, en un extremo del contenedor. Las características físicas e hidráulicas del polvo de piedra utilizado como sustrato, se presentan en la Tabla 1 (UNE-EN-13041 2007).

Tabla 1. Características físicas del polvo de piedra utilizado como sustrato en el experimento con el cultivo de culantro coyote hidropónico.

\begin{tabular}{|c|c|c|c|}
\hline \multirow{2}{*}{ Parámetro } & \multicolumn{2}{|c|}{ Rangos óptimos ${ }^{1}$} & \multirow{2}{*}{$\begin{array}{c}\text { Polvo } \\
\text { de piedra }\end{array}$} \\
\hline & Mínimo & Máximo & \\
\hline Densidad aparente $\left(\mathrm{g} \cdot \mathrm{ml}^{-1}\right)$ & 0,15 & $<0,15$ & 1,14 \\
\hline Densidad real (g.ml $\left.\mathrm{m}^{-1}\right)$ & $<1,0$ & 1,0 & 2,4 \\
\hline Porosidad total $\left(\mathrm{cm} \mathrm{cm}^{-3}\right)$ & $>0,85$ & & 52,5 \\
\hline Humedad (\%) capacidad de contenedor (CC) $1 \mathrm{kPa}$ & 55 & 70 & 19,50 \\
\hline Humedad (\%) punto marchitez permanente (PMP) $10 \mathrm{kPa}$ & 25 & 30 & 9,6 \\
\hline Aireación (\%) a CC (1 kPa) & 15 & 30 & 33,0 \\
\hline Agua total disponible (\%CC - \%PMP) & 24 & 40 & 9,90 \\
\hline
\end{tabular}

1 Adaptado de Ansorena (1994)

Se utilizó semilla de la variedad local de Tres Equis, Turrialba, la cual fue limpiada, seleccionada y desinfectada por inmersión (5 minutos) en una solución de TCMTB (benzotiazol: $1 \mathrm{ml} \cdot \mathrm{L}^{-1}$ ). El trasplante se realizó a los 60 días de edad con aproximadamente 7 hojas verdaderas a una densidad de $7 \mathrm{~cm}$ x $7 \mathrm{~cm}\left(49\right.$ plantas. $\left.\mathrm{m}^{-2}\right)$.

El sistema de ambiente protegido consistió en la colocación de una cobertura de protección de sarán o plástico sobre sobre arcos de metal $(12,5 \mathrm{~mm})$ de $1,5 \mathrm{~m}$ de ancho, fijados a la cama de cultivo, con una altura de $1,2 \mathrm{~m}$ sobre el cultivo y separados cada $\mathrm{m}$. El estudio comprendió 2 etapas consecutivas, de 50 días cada una, durante un mismo ciclo de cultivo. En etapa I, en época de verano, cada bancal de cultivo fue protegido con sarán $30 \%$ de sombra desde el trasplante hasta la primera cosecha. En etapa II, en época de invierno, el cultivo fue protegido con techo plástico $(50 \mu \mathrm{m})$ desde el primer corte hasta la segunda cosecha. En ambas etapas se aplicaron 4 tratamientos que combinaron 2 condiciones ambientales: un cultivo protegido $(\mathrm{CP})$ y otro sin protección (SP) con 2 niveles de nutrición: alta (NA) y baja concentración de nutrientes (NB) en solución nutritiva (Tabla 2). 
Tabla 2. Descripción de tratamientos utilizados en el experimento, para la protección del cultivo de culantro coyote hidropónico durante la etapa I en época seca con sarán rojo y etapa II en época lluviosa con techo plástico.

\begin{tabular}{|c|c|c|c|}
\hline Etapa y fecha & Tratamiento (siglas) & Condición de luz & Nutrición \\
\hline \multirow{4}{*}{$\begin{array}{l}\text { Etapa I: } \\
\text { época de verano } \\
\text { del } 18 / 04 \text { al } 12 / 06 / 2016\end{array}$} & Con protección y nutrición alta & $30 \%$ sombra $(\mathrm{CP})$ & Alta (NA) \\
\hline & Con protección y nutrición baja & $30 \%$ sombra $(\mathrm{CP})$ & Baja (NB) \\
\hline & Sin protección y nutrición alta & Pleno sol (SP) & Alta (NA) \\
\hline & Sin protección y nutrición baja & Pleno sol (SP) & Baja (NB) \\
\hline \multirow{4}{*}{$\begin{array}{l}\text { Etapa II: } \\
\text { época de invierno } \\
\text { del 13/06 al 07/08/2016 }\end{array}$} & Con protección y nutrición alta & Techo plástico $(\mathrm{CP})$ & Alta (NA) \\
\hline & Con protección y nutrición baja & Techo plástico (CP) & Baja (NB) \\
\hline & Sin protección y nutrición alta & Plena lluvia (SP) & Alta (NA) \\
\hline & Sin protección y nutrición baja & Plena lluvia (SP) & Baja (NB) \\
\hline
\end{tabular}

Los tratamientos con baja (NB) y alta concentración (NA) de nutrientes (Tabla 3) fueron definidos considerando los rangos habitualmente utilizados para la producción hidropónica de vegetales de hojas (Urrestarazu 2015, Resh 2001, Soto y Ramírez 2005, Rodríguez et al. 2001).

Tabla 3. Concentraciones utilizadas en los tratamientos con baja (NB) y alta (NA) concentración de nutrientes en el experimento, en cultivos protegidos de culantro coyote hidropónico durante la etapa I en época seca con sarán rojo y etapa II en época lluviosa con techo plástico.

\begin{tabular}{|c|c|c|c|c|c|c|c|c|c|c|c|c|c|}
\hline \multirow{2}{*}{$\begin{array}{c}\text { Tratamiento } \\
\text { de } \\
\text { nutrición }\end{array}$} & \multicolumn{11}{|c|}{ Concentración (mg.L $\left.\mathrm{L}^{-1}\right)$} & \multicolumn{2}{|r|}{$\mathrm{CE}$} \\
\hline & $\mathrm{N}$ & $\mathrm{P}$ & $\mathrm{K}$ & $\mathrm{Mg}$ & $\mathrm{S}$ & $\mathrm{Ca}$ & $\mathrm{Fe}$ & $\mathrm{Cu}$ & $\mathrm{Mn}$ & $\mathrm{Zn}$ & $\mathrm{B}$ & Mo & $\mu \mathrm{S} . \mathrm{cm}^{-1}$ \\
\hline Baja (NB) & 75 & 17 & 100 & 18 & 32 & 81 & 0,4 & 0,1 & 0,2 & 0,1 & 0,2 & 0,04 & 0,8 \\
\hline Alta (NA) & 225 & 52 & 300 & 53 & 97 & 225 & 2,0 & 0,5 & 1,0 & 0,5 & 1,0 & 0,20 & 2,3 \\
\hline
\end{tabular}

Los 4 tratamientos fueron distribuidos en un diseño de 5 bloques con parcelas divididas, con arreglo bifactorial $2 \times 2$, para un total de 20 unidades experimentales. La parcela principal correspondió a la condición de protección y la subparcela al nivel de nutrición. El área de la unidad de muestreo semanal fue de $3 \mathrm{~m}^{2}$ para variables de crecimiento y de $1 \mathrm{~m}^{2}$ para variables de riego y de rendimiento.

Se utilizó un sistema de riego localizado, con cintas de goteo cada $20 \mathrm{~cm}\left(0,8 \mathrm{~L} \cdot \mathrm{h}^{-1}\right)$, y separadas a $7 \mathrm{~cm}$ coincidentes con cada hilera de cultivo. La demanda hídrica del cultivo $\left(\mathrm{L} \cdot \mathrm{m}^{-2} \cdot \mathrm{dí}^{-1}\right)$ fue estimada con i- un $15 \%$ de agotamiento del agua total disponible (Tabla 1) en ii- el volumen de sustrato $\left(100 \mathrm{~L}^{-1} \cdot \mathrm{m}^{-2}\right)$ y iii- aproximadamente un $20 \%$ de drenaje preestablecido. Una vez determinado el requerimiento de agua $\left(\mathrm{L} \cdot \mathrm{m}^{-2}\right.$. día $\left.\mathrm{a}^{-1}\right)$, se calculó el tiempo de riego a partir del número de goteros por $\mathrm{m}^{-2} \mathrm{y}$ del caudal promedio por gotero. Para el manejo del riego, el volumen de riego estimado fue distribuido durante el día en riegos cortos, cuya frecuencia fue ajustada según el porcentaje de drenaje estimado diariamente como el cociente entre volúmenes de drenaje y aportes de agua. El volumen de drenaje por repetición fue recolectado diariamente en un recipiente plástico $(20 \mathrm{~L})$ y medido a las 6 a.m. antes del inicio del primer riego. 
Durante el experimento, la precipitación (PP) fue medida con 4 pluviómetros, 2 en los tratamientos CP y 2 en los cultivos SP. En ambas etapas, los aportes de riego (R) fueron similares en ambas condiciones de protección y de nutrición (Tabla 4). Sin embargo, en los cultivos a la intemperie (SP), debido a la precipitación (PP), el aporte total de agua $(\mathrm{R}+\mathrm{PP})$ fue ligeramente mayor en etapa I de época seca y apreciablemente superior en etapa II de época de invierno.

Tabla 4. Volúmenes $\left(\mathrm{L}_{\mathrm{m}} \mathrm{m}^{-2}\right)$ de riego $(\mathrm{R})$, precipitación $(\mathrm{PP})$, riego + precipitación $(\mathrm{R}+\mathrm{PP})$ y drenaje, en etapa I en época seca y etapa II en época lluviosa de un cultivo de culantro coyote hidropónico, bajo condición protegida (CP), sin protección (SP), con nutrición baja (NB) y nutrición alta (NA). Alajuela, Costa Rica. 2016.

\begin{tabular}{|c|c|c|c|c|c|}
\hline Etapa & Tratamiento & Riego & PP & $\mathrm{R}+\mathrm{PP}$ & Drenaje \\
\hline \multirow{4}{*}{$\begin{array}{l}\text { Etapa I: época de verano del } 18 / 04 \text { al } \\
12 / 06 / 2016 \text { protegido con sarán }\end{array}$} & Con protección $(\mathrm{CP})$ & 237 & 249 & 485 & 315 \\
\hline & Sin protección (SP) & 237 & 298 & 535 & 314 \\
\hline & Nutrición baja (NB) & 237 & 274 & 510 & 301 \\
\hline & Nutrición alta (NA) & 237 & 274 & 510 & 308 \\
\hline \multirow{4}{*}{$\begin{array}{l}\text { Etapa II: época de invierno del } 13 / 06 \text { al } \\
07 / 08 / 2016 \text { protegido con techo plástico }\end{array}$} & Con protección (CP) & 319 & 0 & 319 & 183 \\
\hline & Sin protección (SP) & 320 & 330 & 650 & 424 \\
\hline & Nutrición baja (NB) & 319 & 165 & 484 & 296 \\
\hline & Nutrición alta (NA) & 319 & 165 & 484 & 300 \\
\hline
\end{tabular}

En etapa I de verano, el volumen de drenaje fue similar entre los cultivos CP y SP; mientras que fue similar en ambos niveles de nutrición. En etapa II de invierno, en el cultivo SP, el volumen de drenaje fue apreciablemente debido a la PP, mientras que fue similar en los dos niveles de nutrición.
La evolución semanal promedio del contenido de $\theta$ en el sustrato, según condición de protección (Figura 1a) y nivel de nutrición (Figura 1b), se mantuvo estable en ambas etapas del ciclo de cultivo, que oscilaron entre valores de 0,30 y $0,45 \mathrm{~cm}^{3} \cdot \mathrm{cm}^{-3}$.
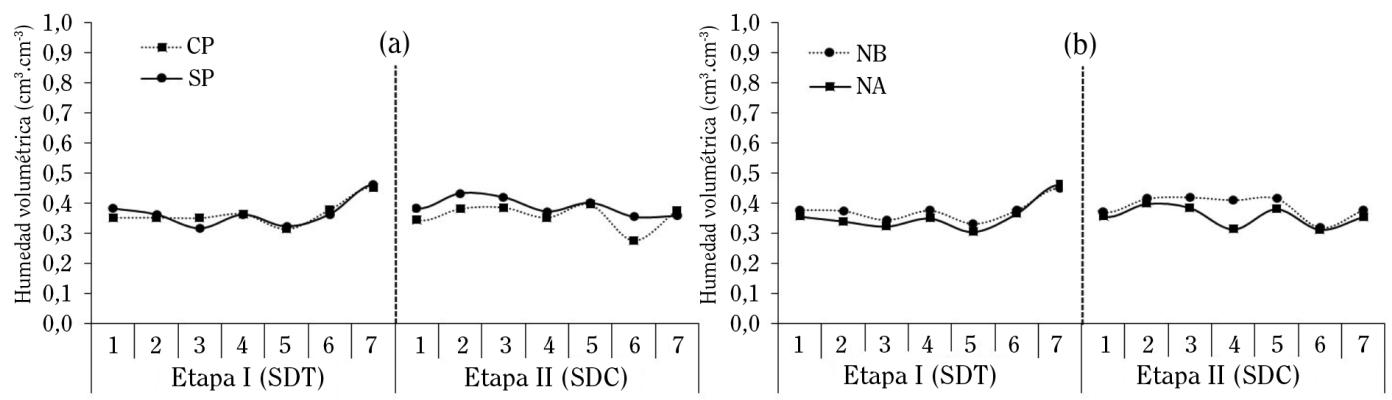

Figura 1. Valores promedio de humedad volumétrica $(\theta)$ bajo (a) protección (CP) y sin (SP) protección, y (b) con baja (NB) y alta (NA) concentración de nutrientes, durante la etapa I en época seca con sarán y etapa II en época lluviosa con techo plástico, de un ciclo de cultivo de culantro coyote hidropónico. Alajuela, Costa Rica. 2016.

Las siglas SDT y SDC indican la semana después del trasplante y después de cosecha, respectivamente. 
Los valores de $\mathrm{CE}$ en el drenaje de los cultivos bajo protección (Figura 2a), fueron similares $\left(1,25 \mathrm{uS} . \mathrm{cm}^{-1}\right)$ en etapa I de época seca bajo sarán y un $47 \%$ superior en la intemperie (SP) en etapa II de época de invierno.
Según nivel de nutrición (Figura 2b), en ambas etapas de cultivo, la CE fue superior con alto nivel de nutrición con valores máximos de $2,62 \mathrm{uS} . \mathrm{cm}^{-1}$ en etapa I y de $2,87 \mathrm{uS} . \mathrm{cm}^{-1}$ en etapa II.
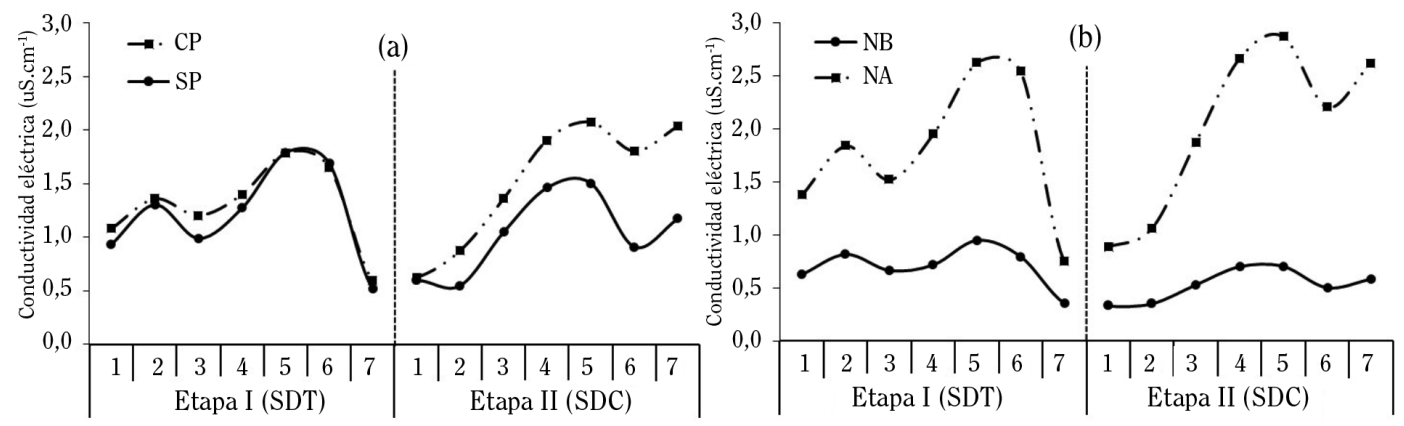

Figura 2. Valores promedio de conductividad eléctrica bajo (a) protección (CP) y sin (SP) protección y (b) con baja (NB) y alta (NA) concentración de nutrientes, durante la etapa I en época seca y etapa II en época lluviosa, de un ciclo de cultivo de culantro coyote hidropónico. Alajuela, Costa Rica. 2016.

Las siglas SDT y SDC indican la semana después del trasplante y después de cosecha, respectivamente.

En cada condición ambiental (CP y SP) se monitoreó la temperatura $\left({ }^{\circ} \mathrm{C}\right)$ y humedad relativa $(\%)$ del aire las cuales se midieron con un sensor de temperatura y humedad relativa; asimismo, se determinaron valores máximos, mínimos y promedios diarios (Tabla 5).

Tabla 5. Valores promedios de temperatura y humedad relativa de máximas, mínimas y promedios, durante la etapa I en época seca y etapa II en época lluviosa, de un cultivo de culantro coyote hidropónico, bajo ambiente protegido (CP) y sin protección (SP). Alajuela, Costa Rica. 2016.

\begin{tabular}{|c|c|c|c|c|c|c|c|}
\hline \multirow{2}{*}{ Etapa } & \multirow{2}{*}{ Ambiente } & \multicolumn{3}{|c|}{ Temperatura $\left({ }^{\circ} \mathrm{C}\right)$} & \multicolumn{3}{|c|}{ Humedad relativa (\%) } \\
\hline & & Máx. & Mín. & Prom. & Máx. & Mín. & Prom. \\
\hline \multirow{2}{*}{ I } & SP & 31,96 & 19,49 & 24,59 & 98,81 & 61,90 & 86,59 \\
\hline & $\mathrm{CP}$ & 32,56 & 19,58 & 24,82 & 98,61 & 60,49 & 85,95 \\
\hline \multirow{2}{*}{ II } & SP & 31,72 & 18,70 & 23,92 & 100,0 & 63,26 & 88,81 \\
\hline & $\mathrm{CP}$ & 31,42 & 18,96 & 23,90 & 99,96 & 63,62 & 88,61 \\
\hline
\end{tabular}

Además, se midió la radiación fotosintéticamente activa (PAR) en el cultivo protegido (CP) y sin protección (SP) con barras provistas de sensores integrados y se determinó la integral de radiación PAR diaria (DLI: mol. $\mathrm{m}^{-2} \cdot$ día $^{-1}$ ), como la sumatoria de los promedios horarios $\left(\mathrm{mol} \cdot \mathrm{m}^{-2} \cdot\right.$ hora $\left.^{-1}\right)$ durante el día (Tabla 6). La DLI fue superior a la intemperie, con valores de 34,2 mol.m ${ }^{-2}$.día ${ }^{-1}$ en SP y 21,2 mol. $\mathrm{m}^{-2}$.día ${ }^{-1}$ en CP en etapa I; y 31,8 mol.m ${ }^{-2}$.día ${ }^{-1}$ en SP y 20,0 mol. $\mathrm{m}^{-2} \cdot$ día $^{-1}$ en CP en etapa II. El déficit de presión de vapor (DPV: $\mathrm{kPa}$ ) se estimó según 
Allen et. al. (2006), mientras que la velocidad del viento (V_V) fue obtenida de la estación del
Instituto Meteorológico Nacional ubicada en la EEFBM (Tabla 6).

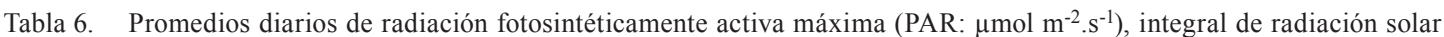

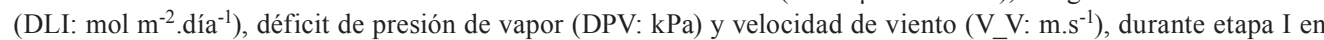
época seca y etapa II en época lluviosa, en un ciclo de cultivo de culantro coyote hidropónico, bajo condiciones con (CP) y sin protección (SP). Alajuela, Costa Rica. 2016.

\begin{tabular}{ccccccccc}
\hline Etapa & Días & PAR CP & PAR SP & DLI CP & DLI SP & DPV CP & DPV SP & V_V \\
\hline & 10 & 584,12 & 921,68 & 11,78 & 19,22 & 1,26 & 2,79 & 2,26 \\
& 20 & 566,43 & 899,04 & 11,57 & 18,84 & 0,98 & 2,65 & 1,92 \\
I (DDS) & 30 & 541,41 & 852,58 & 11,38 & 18,41 & 1,18 & 2,78 & 1,77 \\
& 40 & 520,24 & 811,91 & 10,38 & 16,23 & 0,97 & 2,71 & 1,05 \\
& 50 & 522,81 & 815,92 & 10,92 & 17,06 & 0,63 & 2,50 & 0,85 \\
\hline \multirow{2}{*}{ II (DDC) } & 10 & 612,26 & 859,97 & 11,64 & 17,41 & 0,76 & 2,65 & 1,02 \\
& 20 & 602,15 & 764,95 & 12,56 & 15,79 & 0,92 & 2,68 & 1,04 \\
& 40 & 551,75 & 805,61 & 10,58 & 15,84 & 0,93 & 2,67 & 1,09 \\
& 559,06 & 839,90 & 11,61 & 18,12 & 0,83 & 2,63 & 1,24 \\
\hline
\end{tabular}

Variables de respuesta. Durante el periodo de investigación se evaluaron parámetros de crecimiento, de uso de agua y de nutrientes del cultivo. En crecimiento, se evalúo la producción de materia seca (MS), la tasa de crecimiento del cultivo (TCC: $\left.\mathrm{g} \cdot \mathrm{m}^{-2} \cdot \mathrm{dí}^{-1}\right)$ e índice de área foliar (IAF: $\mathrm{cm}^{2} . \mathrm{cm}^{-2}$ ); en uso de agua la evapotranspiración del cultivo $\left(\mathrm{ET}_{\mathrm{c}}\right.$ : L. $\mathrm{m}^{-2}$ ) y en uso de nutrientes se evaluó la concentración de absorción (CA: mg. $\mathrm{L}^{-1}$ ), la tasa de absorción (TA: g.m ${ }^{2} \cdot$ día $^{-2}$ ), la absorción y lixiviación total $\left(\mathrm{g}^{-\mathrm{m}^{-2}}\right)$ y la eficiencia de recuperación (ER: g. $\left.\mathrm{g}^{-1}\right)$.

Para determinar el contenido de MS y el IAF en cada tratamiento, cada 10 días se recolectaron 5 plantas (macollas)/repetición hasta los 50 días donde se analizaron 20 plantas/repetición, utilizando un horno de ventilación forzada a $72^{\circ} \mathrm{C}$ hasta peso constante. El área foliar (AF: $\mathrm{cm}^{2}$ ) por planta (macolla) se obtuvo con un medidor de AF (LI-COR Mod. LI-3100C) y se estimó el índice de área foliar (IAF: $\mathrm{m}^{2} \cdot \mathrm{m}^{-2}$ ).

La cantidad total de nutrientes extraídos por tratamiento se determinó para periodos de 10 días, a partir de contenido de MS (g.m $\left.\mathrm{m}^{-2}\right)$ y su respectiva concentración de nutrientes $(\%$, mg. $\mathrm{kg}^{-1}$ ), en una muestra combinada de MS obtenida desde las 5 repeticiones/tratamiento. Según Bertsch (2003), el coeficiente de variación de la concentración de nutrientes entre repeticiones presenta valores menores a $15 \%$, por lo cual las muestras de MS se pueden fundir en una sola, ya que efectuar los análisis individuales para cada repetición incrementa los costos. La absorción de nutrientes fue graficada en función del tiempo cronológico (días) y en grados centígrados. día ${ }^{-1}\left({ }^{\circ} \mathrm{Cd}\right)$ según (Murray 2008). Se asumió una temperatura base de $10^{\circ} \mathrm{C}$ para cultivos de climas cálidos (Krug 1999). 
La TCC $\left(\mathrm{g} \mathrm{m}^{-2} \cdot\right.$ día $\left.^{-1}\right)$ se determinó a partir de la masa de MS $\left(\mathrm{m}=\mathrm{g} . \mathrm{m}^{-2}\right)$ producida entre el tiempo en días $\left(\mathrm{t}_{1}\right)$ del muestreo $1\left(\mathrm{~m}_{1}\right)$ y el muestreo $2\left(\mathrm{~m}_{2} \mathrm{y} \mathrm{t}_{2}\right)$, según la ecuación $\mathrm{TCC}=\mathrm{m}_{2}-\mathrm{m}_{1} / \mathrm{t}_{2}-\mathrm{t}_{1}$. La tasa de absorción de nutrientes $\left(\mathrm{TA}=\mathrm{g} \cdot \mathrm{m}^{-2} \cdot \mathrm{día}^{-1}\right)$ se calculó a partir de la cantidad de nutriente absorbido $\left(\mathrm{N}_{\mathrm{ab}}=\mathrm{g} \cdot \mathrm{m}^{-2}\right)$ en el tiempo días del muestreo $1\left(\mathrm{~N}_{\mathrm{ab} 1} \mathrm{y} \mathrm{t}_{1}\right)$ y el muestreo $2\left(\mathrm{~N}_{\mathrm{ab} 2 \text { y }} \mathrm{t}_{2}\right)$ según la ecuación $\mathrm{TA}=\mathrm{N}_{\mathrm{ab} 2}-\mathrm{N}_{\mathrm{ab} 1} / \mathrm{t}_{2}-\mathrm{t}_{1}$.

La concentración de absorción de nutrientes (CA: mg.L $\mathrm{L}^{-1}$ ) se estimó como el cociente entre nutriente absorbido $\left(\mathrm{mg} \cdot \mathrm{m}^{-2}\right)$ y la $\mathrm{ET}_{\mathrm{c}}$ $\left(\mathrm{L}_{\mathrm{m}} \mathrm{m}^{-2}\right.$ ) (Sonneveld 2002). La $\mathrm{ET}_{\mathrm{c}}$ se calculó por el método de balance de agua en el sustrato (Soto 2018), a partir de los volúmenes de agua aportada (riego $(\mathrm{R})+$ precipitación $(\mathrm{P})$, de drenaje (D) y de la diferencia entre los contenidos de humedad del sustrato $\left(\Delta \theta=\theta_{\mathrm{i}}-\theta_{\mathrm{f}}\right)$ al inicio $\left(\theta_{\mathrm{i}}\right)$ y al final $\left(\theta_{\mathrm{f}}\right)$ de un periodo de 10 días $\left(\mathrm{ET}_{\mathrm{c}}=\mathrm{R}+\mathrm{P}-\mathrm{D} \pm \Delta \theta\right)$. El volumen de riego aplicado $\left(\mathrm{L} . \mathrm{m}^{-2}\right)$ se estimó a partir del tiempo de riego, el número de goteros $/ \mathrm{m}^{2}$ y el caudal promedio/gotero. El drenaje y el contenido de $\theta$ de cada repetición se midió diariamente a las 6 a.m. antes de iniciar el primer riego. La $\theta$ en el sustrato se midió con un sensor de capacitancia (Mod. GS3, DECAGON DEVICES).

La concentración de nutrientes, en solución nutritiva y en drenaje se determinó cada 10 días, en muestras compuestas de las repeticiones de cada tratamiento, a partir de sub-muestras recolectadas diariamente. Las cantidades de nutriente aplicado $\left(\mathrm{g} . \mathrm{m}^{-2}\right)$ y lixiviado $\left(\mathrm{g} . \mathrm{m}^{-2}\right)$ se obtuvieron como el producto de los volúmenes de riego y drenaje $\left(\mathrm{L}^{\mathrm{m}} \mathrm{m}^{-2}\right)$ por sus respectivas concentraciones de macro (g.L $\left.\mathrm{L}^{-1}\right)$ y micronutrientes $\left(\mathrm{mg} . \mathrm{L}^{-1}\right)$. La eficiencia de recuperación $\left(\mathrm{ER}=\mathrm{g}_{\mathrm{g}} \mathrm{g}^{-1}\right)$ se determinó como el cociente entre nutriente absorbido $\left(\mathrm{N}_{\mathrm{ab}}=\mathrm{g} . \mathrm{m}^{-2}\right)$ y nutriente aplicado (g.m²), según Doberman (2005).

Análisis estadístico. Los datos que fueron normales y homocedásticos fueron sometidos a un análisis de varianza (ANDEVA), por medio del software estadístico InfoStat versión 2016, para determinar si hubo efecto $(p<0,05)$ de la interacción o efectos individuales de la condición de protección y/o el nivel de nutrición. En el ANDEVA se utilizó un modelo para bloques al azar con parcelas divididas según Di Rienzo et al. (2012).

$$
\mathrm{Y}_{\mathrm{ijk}}=\mu+\tau_{\mathrm{i}}+\beta_{\mathrm{j}}+(\tau \beta)_{\mathrm{ij}}+\gamma \mathrm{ijk}+(\tau \gamma)_{i k}+\varepsilon_{\mathrm{ijk}}
$$

Donde:

$\mathrm{Y}_{\mathrm{ijk}}=$ representa la observación en el k-ésimo nivel del factor aplicado a la subparcela, de la i-ésima parcela principal en el j-ésimo bloque.

$\mu \quad=$ media general.

$\mathrm{G}_{\mathrm{i}}=$ el efecto del i-ésimo nivel del factor aplicado a la parcela principal.

$\beta_{\mathrm{j}} \quad=$ el j-ésimo bloque.

$(\mathrm{T} \beta)_{\mathrm{ij}}=$ el error experimental de las parcelas principales que se simboliza como la interacción entre el factor principal y los bloques. $\gamma \mathrm{ijk}=$ el efecto del k-ésimo nivel del factor asociado a la subparcela dentro de la i-ésima parcela principal del j-ésimo bloque.

$(\tau \gamma)_{\mathrm{ik}}=$ la interacción del factor principal con el factor aplicado a las subparcelas.

$\mathrm{E}_{\mathrm{ijk}}=\mathrm{el}$ error experimental a nivel de subparcelas.

\section{RESULTADOS}

En el presente estudio, no hubo efecto de la interacción entre los factores protección y nutrición $(p>0,05)$ sobre el crecimiento (MS, TCC, IAF), la absorción total, tasa de absorción, lixiviación, ER, y CA de nutrientes. 
En ambas etapas del experimento, el crecimiento del cultivo (MS, TCC; IAF) fue afectado $(\mathrm{p}<0,05)$ por la condición de protección y el nivel de nutrición, ya que fue superior en condiciones protegidas y con alto nivel de nutrientes, con un efecto de mayor magnitud debido al factor nutrición (Figura 3). El comportamiento del crecimiento expresado en MS e IAF, en cuanto a magnitud y tendencia, fue diferente en las 2 etapas del cultivo. En la primera etapa del experimento, en época de verano, se observa un ligero crecimiento hasta $30 \mathrm{DDT}$, luego incrementa exponencialmente con una tendencia creciente aun al momento de la cosecha. En etapa II, el crecimiento (MS, IAF), prácticamente, se duplicó respecto a la etapa I, con una tendencia a incrementar desde el inicio y a disminuir a partir los 40 DDC.

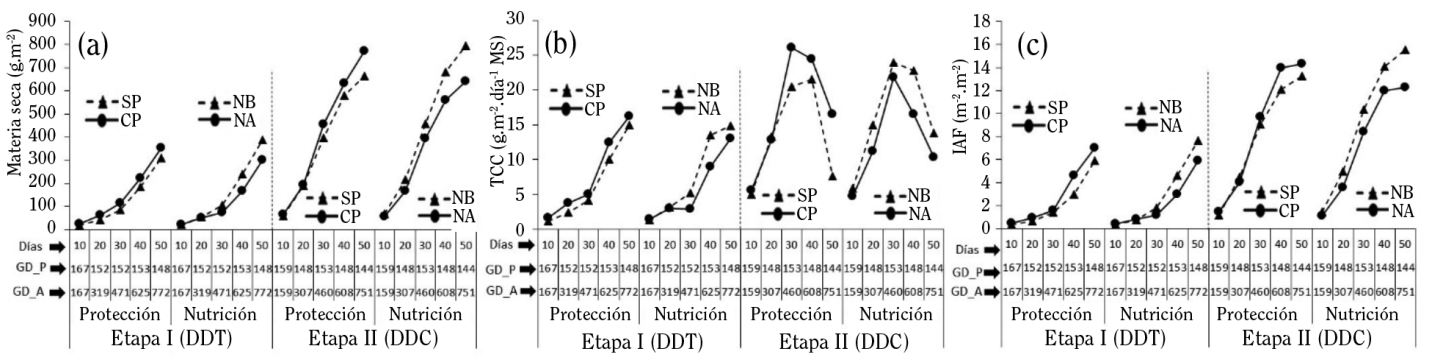

Figura 3. Efecto de la protección (CP: con protección y SP: sin protección) y la nutrición (NA: nutrición alta y NB: nutrición baja) sobre la (a) producción de materia seca (MS), (b) la tasa de crecimiento (TCC) y (c) el índice de área foliar (IAF), durante la etapa I en época seca y etapa II en época lluviosa de un ciclo de cultivo de culantro coyote hidropónico, según tiempo cronológico (días) y tiempo térmico en grados centígrados día $\left({ }^{\circ} \mathrm{C} \cdot \mathrm{d}^{-1}\right)$ por periodo de 10 días (GD_P) y acumulado (GD_P). Alajuela, Costa Rica. 2016.

Las siglas SDT y SDC indican la semana después del trasplante y después de cosecha, respectivamente.

Del comportamiento obtenido en MS, el IAF fue consecuente con el patrón observado en la TCC en ambas etapas del cultivo (Figura 3c), la cual incrementó sustancialmente a partir de 30 DDT en etapa I, y desde el inicio DDC en etapa II, alcanzando valores máximos entre los 30 y 40 DDC así como una disminución hacia el final de la etapa (Figura 3b).

Evapotranspiración y absorción de nutrientes. A partir de la relación de estos parámetros, se determinó la concentración de absorción (CA: mg. $\mathrm{L}^{-1}$ ) de nutrientes, lo cual representa las curvas de absorción en cultivos hidropónicos como CA en mg de nutrientes por $\mathrm{L}$ de ETc, análogo, aunque no lo mismo, a las curvas de absorción en cultivo en suelo (kg.ha ${ }^{-1}$ ). En las 2 etapas del cultivo de verano, en invierno, el comportamiento en la tasa de $\mathrm{ET}_{\mathrm{c}}$ (Figura 4a: L. $\mathrm{m}^{-2} \cdot$ día $^{-1}$ ) fue consecuente con la tendencia observada en la acumulación de MS, IAF, TCC. Tanto la $\mathrm{ET}_{\mathrm{c}}$ diaria como la $\mathrm{ET}_{\mathrm{c}}$ total (Figura $4 \mathrm{~b}$ : L. $\mathrm{m}^{-2}$ ), fueron en promedio un $43 \%$ inferior en el cultivo protegido. Por otra parte, al considerar el nivel de nutrición, se observó que en ambas etapas del experimento las diferencias entre valores de $\mathrm{ET}_{\mathrm{c}}$ fueron leves, con un promedio de $4 \%$ superior cuando el nivel de nutrición fue alto. 

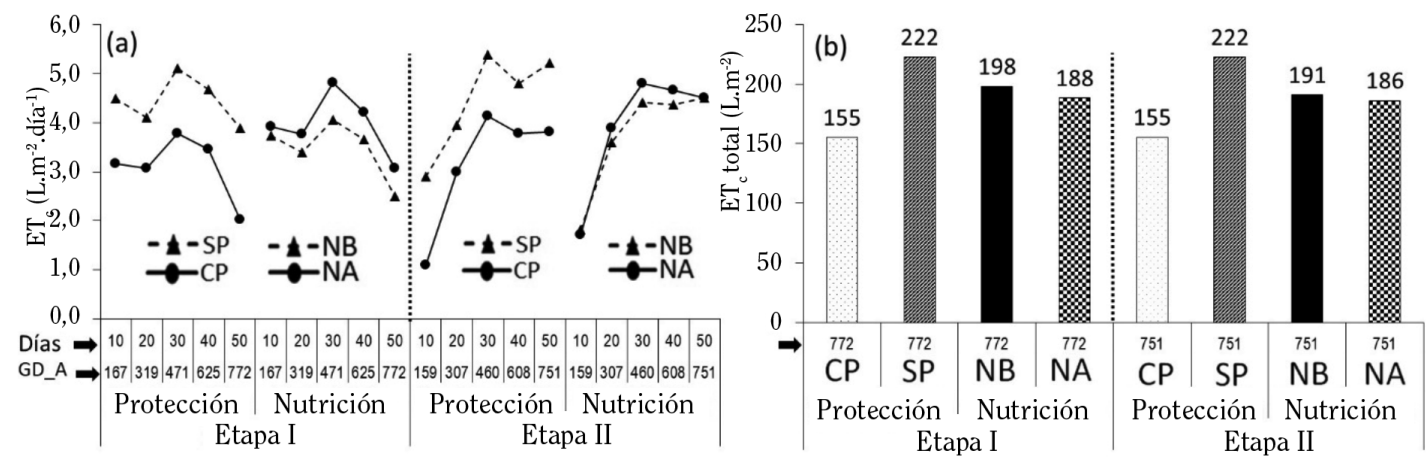

Figura 4. Evapotranspiración del cultivo $\left(E T_{c}\right)$ (a) promedio diario $\left(\mathrm{L} \mathrm{m}^{-2} \cdot \mathrm{dí}^{-1}\right)$ y (b) total $\left(\mathrm{L} \cdot \mathrm{m}^{-2}\right)$ en etapa I en época seca y etapa II en época lluviosa, en un ciclo de cultivo de culantro coyote hidropónico bajo protección (CP), sin protección (SP), con nutrición baja (NB) y nutrición alta (NA). Alajuela, Costa Rica. 2016.

En ambas etapas del experimento, bajo protección con sarán en época de verano y bajo techo plástico en época lluviosa, la CA de la mayoría de los nutrientes fue afectada $(\mathrm{p}<0,05)$ individualmente por ambos factores (Tabla 7).

Tabla 7. Análisis de varianza que muestra la interacción o los efectos individuales de la condición de protección $(\mathrm{P})$ y del nivel de nutrición $(\mathrm{N})$ sobre la concentración de absorción (CA) de nutrientes.

\begin{tabular}{|c|c|c|c|c|c|c|c|c|c|c|c|c|c|c|c|c|c|c|c|c|c|c|c|c|c|}
\hline \multirow{2}{*}{\multicolumn{2}{|c|}{ Efecto }} & \multicolumn{13}{|c|}{ Etapa I en verano } & \multicolumn{11}{|c|}{ Etapa II en invierno } \\
\hline & & $\mathrm{N}$ & $\mathrm{P}$ & $\mathrm{K}$ & $\mathrm{C}$ & M & & $S$ & $\mathrm{Fe}$ & $\mathrm{Mn}$ & $\mathrm{Cl}$ & Z & & B & $\mathrm{N}$ & $\mathrm{P}$ & K & $\mathrm{Ca}$ & $\mathrm{Mg}$ & S & $\mathrm{Fe}$ & $\mathrm{Mn}$ & $\mathrm{Cu}$ & $\mathrm{Zn}$ & $\mathrm{B}$ \\
\hline \multicolumn{26}{|c|}{$\mathrm{P} \times \mathrm{N}$} \\
\hline \multirow[t]{2}{*}{ త } & $\mathrm{P}$ & $*$ & * & * & * & * & & * & $*$ & $*$ & * & 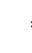 & & $*$ & $*$ & $*$ & $*$ & $*$ & $*$ & $*$ & * & $*$ & $*$ & * & $*$ \\
\hline & $\mathrm{N}$ & $*$ & $*$ & * & * & * & & * & $*$ & $*$ & $*$ & & & $*$ & $*$ & $*$ & $*$ & & & $*$ & $*$ & $*$ & $*$ & $*$ & $*$ \\
\hline
\end{tabular}

* indica interacción o efectos independientes $(\mathrm{p}<0,05)$ de la condición de protección y del nivel de nutrición.

El comportamiento de la $\mathrm{ET}_{\mathrm{c}}$ fue consecuente con el patrón de crecimiento del cultivo (MS, TCC e IAF), lo cual a su vez correspondió al comportamiento observado y la CA (Figura 5). En ambas etapas de cultivo, hubo mayor CA bajo condición de cultivo protegido y cuando el nivel de nutrición fue alto. La magnitud del incremento en la CA al pasar de un cultivo SP a otro CP, y de un nivel de nutrición bajo a otro alto, fue similar en ambas etapas. Sin embargo, independientemente de la condición de protección y del nivel de nutrición, los valores de CA fueron superiores en la segunda etapa experimental, donde hubo mayor producción de biomasa (MS). 


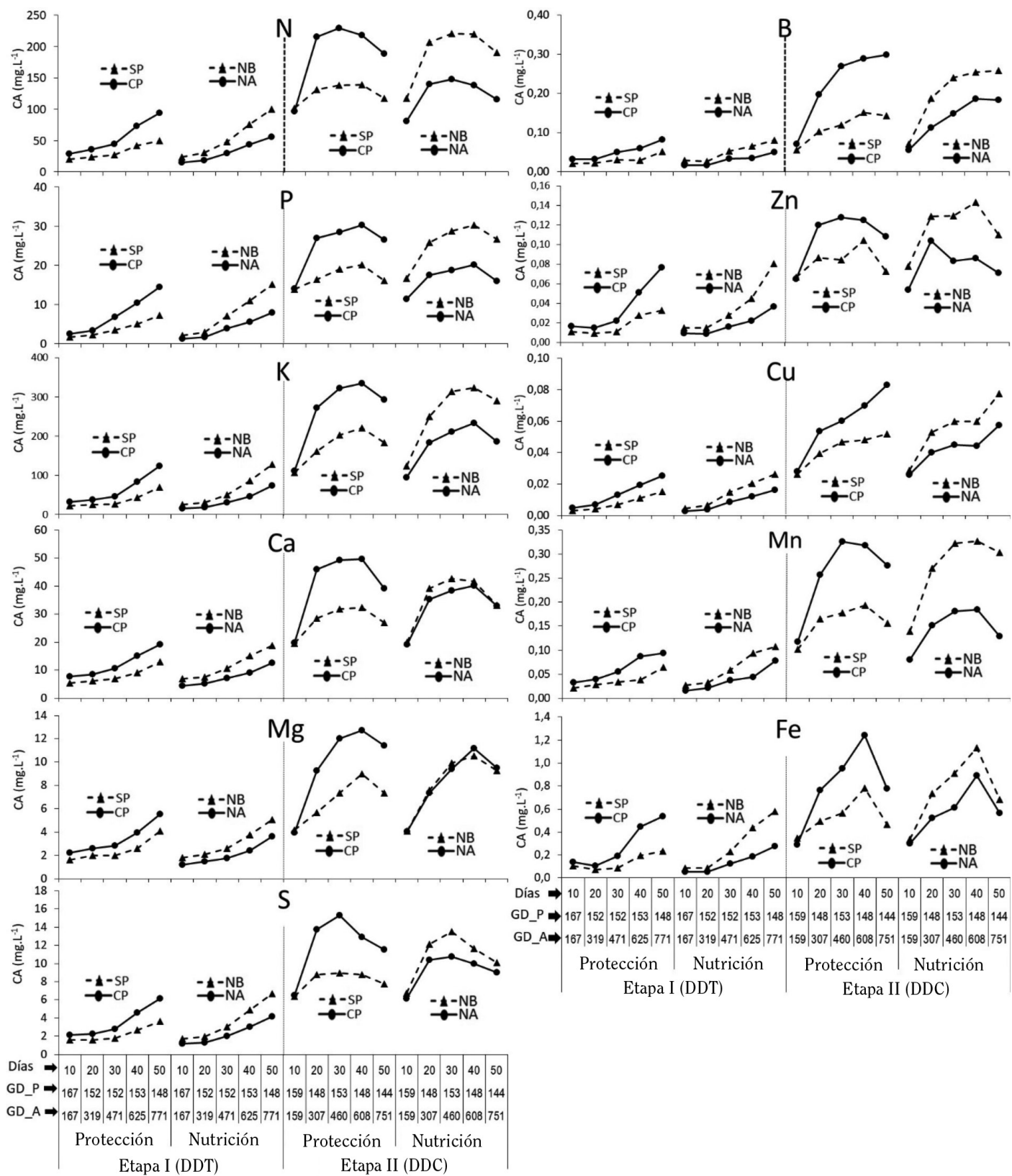

Figura 5. Efecto de la protección $(\mathrm{CP}=$ con protección y $\mathrm{SP}=\sin$ protección $)$ y la nutrición $(\mathrm{NA}=$ nutrición alta y $\mathrm{NB}=$ nutrición baja) sobre la concentración de absorción (CA) de nutrientes, durante la etapa I en época seca y etapa II en época lluviosa de un ciclo de cultivo de culantro coyote hidropónico, según tiempo cronológico (días) y grados centígrados día $\left({ }^{\circ} \mathrm{C} . \mathrm{d}^{-1}\right)$ por periodo de 10 días (GD_P) y acumulados (GD_P). Alajuela, Costa Rica. 2016. Las siglas SDT y SDC indican la semana después del trasplante y después de cosecha, respectivamente. 
En etapa I de verano, la CA para todos los nutrientes incrementó ligeramente en los primeros 30 DDT, posteriormente, tuvo una tendencia creciente consecuente con el comportamiento en el crecimiento del cultivo. Las diferencias promedio en CA, entre cultivos CP y SP, fluctuaron desde un máximo de $100 \%$ en el caso del Fe hasta un $54 \%$ en el S. Respecto a los efectos del nivel de nutrición, dichas diferencias oscilaron desde $99 \%$ en el caso del Zn hasta un $63 \%$ para el S.

En la etapa II, la CA fue superior a la etapa I, e incrementó de forma exponencial desde el inicio del cultivo posterior a la primera cosecha, alcanzó valores máximos entre los 30 y 40 DDC y disminuyó hacia el final de la etapa. Las diferencias promedias en CA, entre cultivos CP y SP, fluctuaron desde un máximo de $77 \%$ para el Mn hasta un $48 \%$ en S. Por otra parte, al considerar el efecto del nivel de nutrición, dichas diferencias oscilaron entre un 3\% para el $\mathrm{Mg}$, y hasta $88 \%$ en el caso del $\mathrm{Mn}$, con la excepción del Ca y el $\mathrm{Mg}$ que no fueron afectados estadísticamente $(\mathrm{p}>0,05)$.

Tasa de absorción (TA) de nutrientes. En condiciones de sombreo con sarán en etapa I de verano y bajo techo plástico en etapa II de invierno, la tasa de absorción fue afectada individualmente por ambos factores $(\mathrm{p}<0,05)$ (Tabla 8). En etapa I de época de verano, la protección afectó la TA del $\mathrm{N}, \mathrm{P}, \mathrm{K}, \mathrm{Fe}, \mathrm{Zn}$ y $\mathrm{B}$, mientras que en etapa II, en invierno, afectó la mayoría de los nutrientes, excepto el $\mathrm{Ca}, \mathrm{Mg}$ y $\mathrm{S}$.

Tabla 8. Análisis de varianza que muestra la interacción o los efectos individuales de la condición de protección $(\mathrm{P})$ y del nivel de nutrición (N) sobre la tasa de absorción (TA) de nutrientes.

\begin{tabular}{|c|c|c|c|c|c|c|c|c|c|c|c|c|c|c|c|c|c|c|c|c|c|c|c|}
\hline \multirow{2}{*}{\multicolumn{2}{|c|}{ Efecto }} & \multicolumn{11}{|c|}{ Etapa I en verano } & \multicolumn{11}{|c|}{ Etapa II en invierno } \\
\hline & & $\mathrm{N}$ & $\mathrm{P}$ & $\mathrm{K}$ & $\mathrm{Ca}$ & $\mathrm{Mg}$ & $\mathrm{S}$ & $\mathrm{Fe}$ & $\mathrm{Mn}$ & $\mathrm{Cu}$ & $\mathrm{Zn}$ & B & $\mathrm{N}$ & $\mathrm{P}$ & $\mathrm{K}$ & $\mathrm{Ca}$ & $\mathrm{Mg}$ & $\mathrm{S}$ & $\mathrm{Fe}$ & $\mathrm{Mn}$ & $\mathrm{Cu}$ & $\mathrm{Zn}$ & B \\
\hline \multirow{3}{*}{ 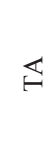 } & $\mathrm{P} \times \mathrm{I}$ & & & & & & & & & & & & & & & & & & & & & & \\
\hline & $\mathrm{P}$ & * & $*$ & * & & & & $*$ & & & * & * & $*$ & $*$ & * & & & & $*$ & * & * & $*$ & * \\
\hline & $\mathrm{N}$ & $*$ & $*$ & * & $*$ & $*$ & $*$ & $*$ & $*$ & $*$ & $*$ & $*$ & $*$ & $*$ & * & & & & $*$ & $*$ & $*$ & $*$ & * \\
\hline
\end{tabular}

* indica interacción o efectos independientes $(\mathrm{p}<0,05)$ de la condición de protección y del nivel de nutrición.

En ambas etapas del experimento, la TA tuvo un comportamiento consecuente con el patrón de crecimiento (MS, IAF, TCC) del cultivo. La mayor TA se presentó cuando el cultivo estuvo protegido y el nivel de nutrición fue alto, y superior en etapa II (Figura 6). En etapa I, la TA aumentó ligeramente en los primeros 10 días, luego incrementó exponencialmente, hasta alcanzar valores máximos a los 40 DDT y disminuyó al final de la etapa. Las diferencias entre TA promedio de cultivos $\mathrm{CP}$ y SP fluctuaron desde un $20 \%$ en el caso del B hasta un $41 \%$ en el Fe. Conforme a los efectos del nivel de nutrición, dichas diferencias oscilaron entre un $28 \%$ para el $\mathrm{Mg}$ hasta el $100 \%$ en el caso del Fe. 


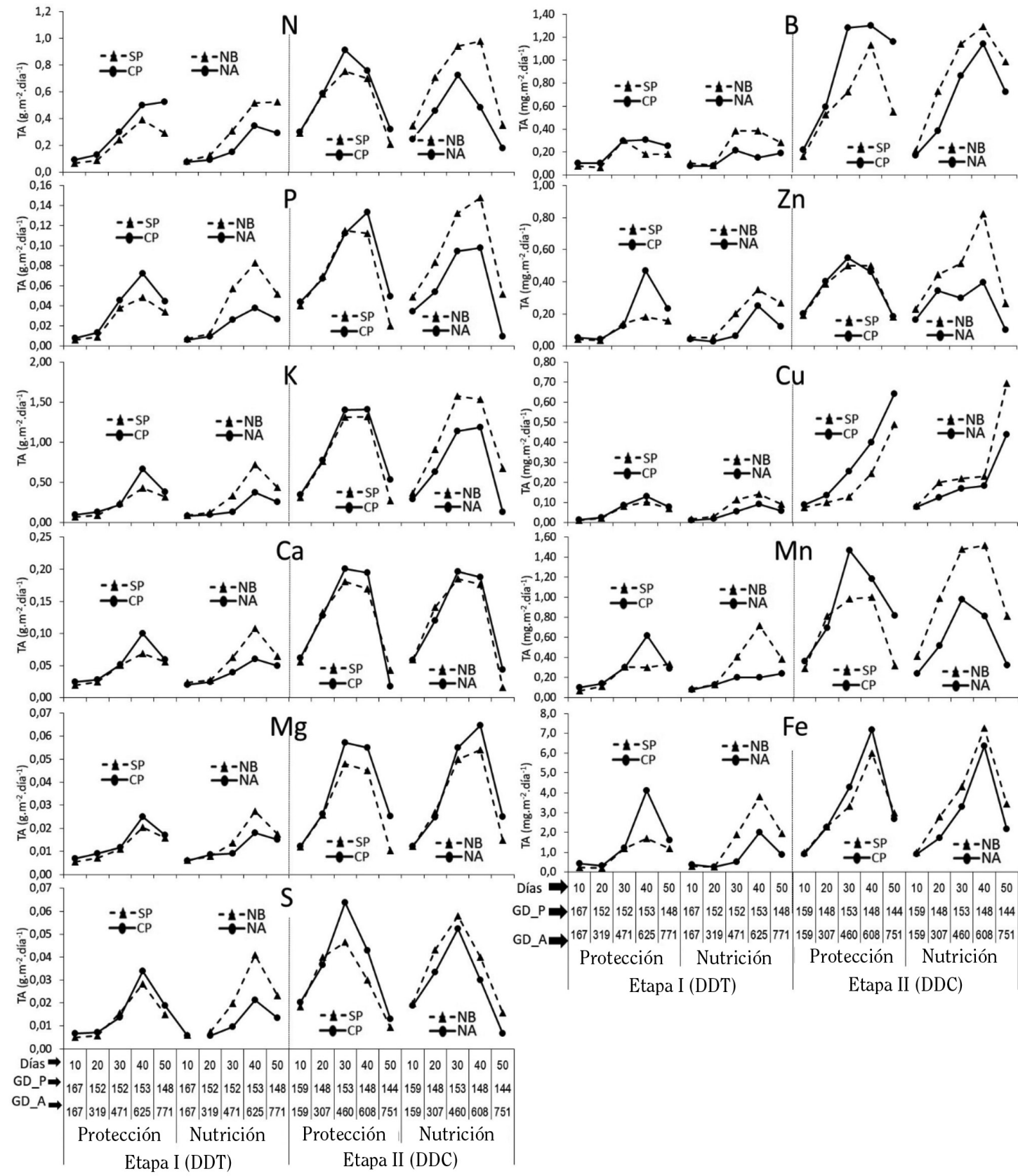

Figura 6. Efecto de la protección $(\mathrm{CP}=$ con protección y $\mathrm{SP}=\sin$ protección) y la nutrición $(\mathrm{NA}=$ nutrición alta y $\mathrm{NB}=$ nutrición baja) sobre la tasa de absorción (TA) de nutrientes, durante la etapa I en época seca y etapa II en época lluviosa de un ciclo de cultivo de culantro coyote hidropónico, según tiempo cronológico (días) y grados centígrados día $\left({ }^{\circ} \mathrm{C} . \mathrm{d}^{-1}\right)$ por periodo de 10 días (GD_P) y acumulados (GD_P). Alajuela, Costa Rica. 2016. 
En etapa II de cultivo, la TA aumentó exponencialmente desde el inicio; alcanzó valores máximos entre los 30 y 40 DDC, y luego disminuyó hacia el final de la etapa (Figura 6). El efecto de la nutrición fue de mayor magnitud que el de la protección. Las diferencias en la TA entre cultivos CP y SP fluctuaron desde un $11 \%$ para el caso de $\mathrm{K}$ hasta un $32 \%$ para el $\mathrm{B}$, mientras que las diferencias entre NA y NB osciló entre un 27\% para el caso del $\mathrm{Cu}$ hasta $81 \%$ para el $\mathrm{Mn}$.

Nutrienteaplicado, absorbidoylixiviado. En ambas etapas de cultivo en época de verano e invierno, la cantidad de nutriente aplicado fue similar en las 2 condiciones de protección, y obviamente, superior cuando el nivel de nutrición fue alto (Figura 7a, 7b).
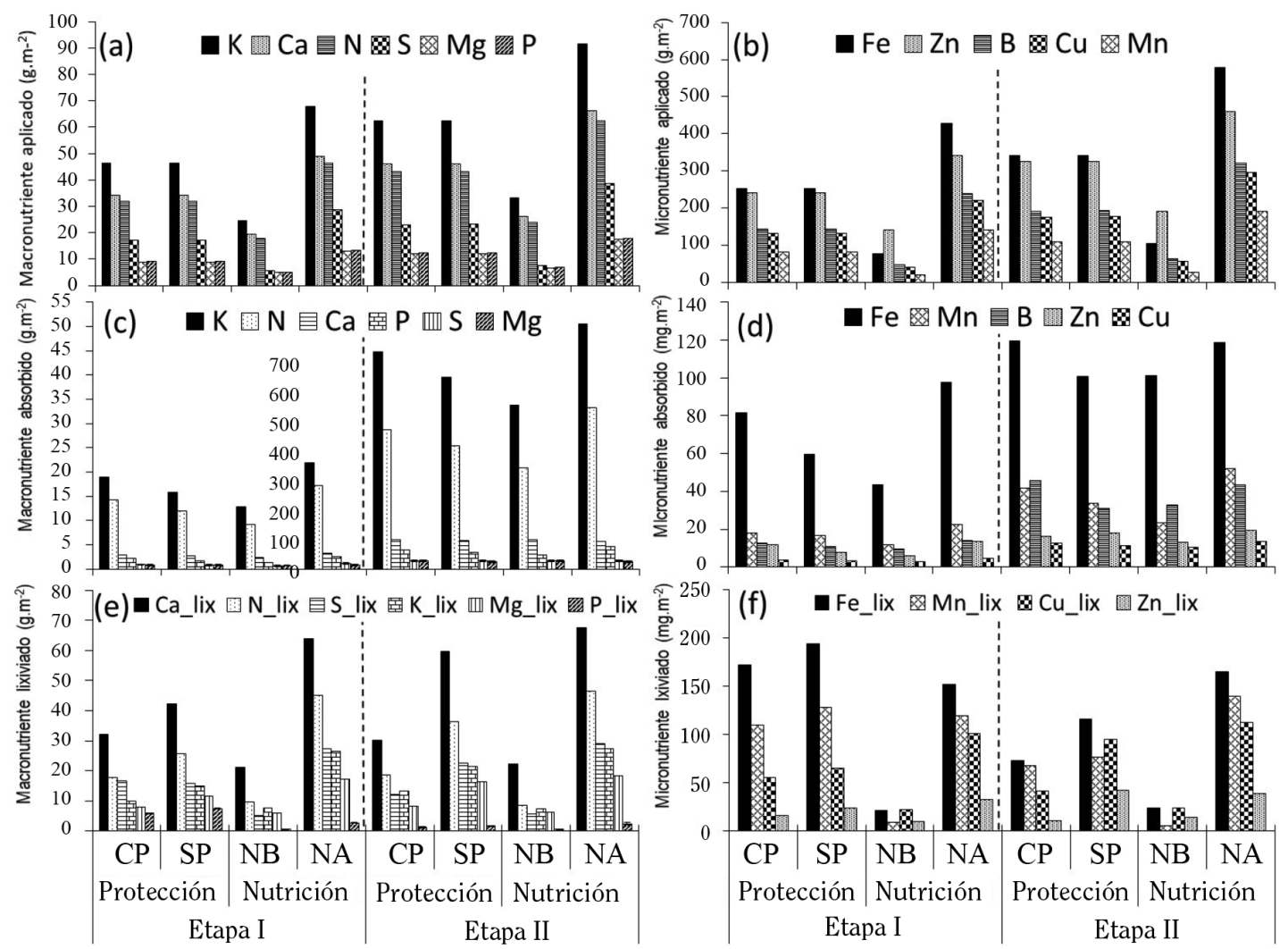

Figura 7. Cantidad total de nutriente aplicado, absorbido y lixiviado; durante la etapa I en época seca y etapa II en época lluviosa de un ciclo de cultivo de culantro coyote hidropónico bajo protección (CP), sin protección (SP), con nutrición baja (NB) y nutrición alta (NA). Alajuela, Costa Rica. 2016.

La cantidad de nutriente absorbido y lixiviado fue afectada $(\mathrm{p}<0,05)$ por la condición de protección y el nivel de nutrición, sin interacción entre factores (Tabla 9). 
Tabla 9. Análisis de varianza que muestra la interacción o los efectos individuales de la condición de protección (P) y del nivel de nutrición $(\mathrm{N})$ sobre el total de absorción y lixiviación de nutrientes.

\begin{tabular}{|c|c|c|c|c|c|c|c|c|c|c|c|c|c|c|c|c|c|c|c|c|c|c|c|}
\hline \multirow{2}{*}{\multicolumn{2}{|c|}{ Efecto }} & \multicolumn{11}{|c|}{ Etapa I en verano } & \multicolumn{11}{|c|}{ Etapa II en invierno } \\
\hline & & $\mathrm{N}$ & $\mathrm{P}$ & K & $\mathrm{Ca}$ & $\mathrm{Mg}$ & $\mathrm{S}$ & $\mathrm{Fe}$ & $\mathrm{Mn}$ & $\mathrm{Cu}$ & $\mathrm{Zn}$ & $\mathrm{B}$ & $\mathrm{N}$ & $\mathrm{P}$ & $\mathrm{K}$ & $\mathrm{Ca}$ & $\mathrm{Mg}$ & $\mathrm{S}$ & $\mathrm{Fe}$ & $\mathrm{Mn}$ & $\mathrm{Cu}$ & $\mathrm{Zn}$ & B \\
\hline \multirow{3}{*}{ 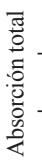 } & $\mathrm{P} \times \mathrm{N}$ & & & & & & & & & & & & & & & & & & & & & & \\
\hline & $\mathrm{P}$ & $*$ & $*$ & $*$ & & & & $*$ & & & $*$ & $*$ & $*$ & $*$ & $*$ & & & & $*$ & $*$ & $*$ & $*$ & * \\
\hline & $\mathrm{N}$ & $*$ & $*$ & $*$ & $*$ & $*$ & $*$ & $*$ & * & $*$ & * & $*$ & $*$ & $*$ & $*$ & & & & $*$ & $*$ & $*$ & $*$ & $*$ \\
\hline \multirow{3}{*}{ 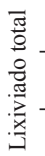 } & $\mathrm{P} \times \mathrm{N}$ & & & & & & & & & & & - & & & & & & & & & & & - \\
\hline & $\mathrm{P}$ & $*$ & $*$ & $*$ & & & & $*$ & $*$ & $*$ & & - & $*$ & $*$ & $*$ & $*$ & $*$ & $*$ & $*$ & $*$ & $*$ & $*$ & - \\
\hline & $\mathrm{N}$ & $*$ & $*$ & $*$ & $*$ & $*$ & $*$ & $*$ & $*$ & $*$ & * & - & $*$ & $*$ & $*$ & $*$ & $*$ & $*$ & $*$ & $*$ & $*$ & $*$ & - \\
\hline
\end{tabular}

* indica interacción o efectos independientes $(\mathrm{p}<0,05)$ de la condición de protección y del nivel de nutrición. - no hubo datos.

Absorción total. Independientemente de ambos factores, en la etapa II de invierno la cantidad total de nutriente absorbido fue muy superior a la etapa I (Figura 7c, 7d). En ambas etapas, indistintamente del nivel de nutrición, la protección del cultivo favoreció la absorción de nutrientes. En etapa I, el sombreo con sarán favoreció la absorción total de N, P, K, Fe, Zn y $\mathrm{B}$; mientras que en la etapa II la protección afectó $(\mathrm{p}>0,05)$ la absorción de la mayoría de los nutrientes, excepto para el $\mathrm{Ca}$, el Mg y el $\mathrm{S}$ (Tabla 9; Figura 7c, 7d).

Al considerar el nivel de nutrición, independientemente de la protección, en ambas etapas del experimento la mayor absorción total se presentó cuando el nivel de nutrición fue alto. En etapa I en verano, el mayor nivel de nutrición favoreció la absorción total de todos los macro y micronutrientes, mientras que en la etapa II, en invierno, únicamente no hubo efecto sobre la absorción de calcio, magnesio y azufre (Tabla 9; Figura 7c, 7d).

En las 2 etapas del experimento, tanto en la condición de protección como de nutrición, el orden decreciente de absorción total de nutrientes fue $\mathrm{K}>\mathrm{N}>\mathrm{Ca}>\mathrm{P}>\mathrm{Mg}>\mathrm{S}>\mathrm{Fe}>\mathrm{Mn}>\mathrm{B}$ $>\mathrm{Zn}>\mathrm{Cu}$ (Figura 7c, 7d).
Lixiviación total. En ambas etapas, en los cultivos con protección, la lixiviación disminuyó, coincidente con una mayor absorción de nutrientes. Por el contrario, al aumentar el nivel de nutrición, aunque la absorción aumentó, simultáneamente incrementó la lixiviación de nutrientes (Figura 7e, 7f).

En la etapa I en época de verano, la protección del cultivo afectó $(\mathrm{p}<0,05)$ la lixiviación de $\mathrm{N}, \mathrm{P}, \mathrm{K}, \mathrm{Fe}, \mathrm{Mn}$ y $\mathrm{Cu}$; mientras que en la etapa II de invierno, ambos factores afectaron $(\mathrm{p}<0,05)$ por igual la lixiviación total de todos los nutrientes (Tabla 9). En la etapa II, en invierno, la mayor cantidad de nutriente lixiviado se presentó en el cultivo a la intemperie y cuando el nivel de nutrición fue alto (Figura 7e, 7f). El calcio fue el elemento que lixivió en mayor cantidad, lo que incrementó su lavado en un $98 \%$ al pasar de una condición protegida a otra sin protección y un $200 \%$ al cambiar de baja a alta concentración de nutrientes.

Eficiencia de recuperación (ER) de nutrientes. En las 2 etapas de cultivo, la ER fue afectada de forma individual por la condición de protección y el nivel de nutrición $(\mathrm{p}<0,05$; Tabla 10). 
Tabla 10. Análisis de varianza que muestra la interacción o los efectos individuales de la condición de protección (P) y del nivel de nutrición $(\mathrm{N})$ sobre la eficiencia de recuperación (ER) de nutrientes.

\begin{tabular}{|c|c|c|c|c|c|c|c|c|c|c|c|c|c|c|c|c|c|c|c|c|c|c|c|}
\hline \multirow{2}{*}{\multicolumn{2}{|c|}{ Efecto }} & \multicolumn{10}{|c|}{ Etapa I en verano } & \multicolumn{12}{|c|}{ Etapa II en invierno } \\
\hline & & $\mathrm{N}$ & $\mathrm{P}$ & K & $\mathrm{Ca}$ & $\mathrm{Mg}$ & $\mathrm{S}$ & $\mathrm{Fe}$ & $\mathrm{Mn}$ & $\mathrm{Cu}$ & $\mathrm{Zn}$ & B & $\mathrm{N}$ & $\mathrm{P}$ & $\mathrm{K}$ & $\mathrm{Ca}$ & $\mathrm{Mg}$ & S & $\mathrm{Fe}$ & $\mathrm{Mn}$ & $\mathrm{Cu}$ & $\mathrm{Zn}$ & B \\
\hline \multicolumn{24}{|c|}{$\mathrm{P} \times \mathrm{N}$} \\
\hline \multirow[t]{2}{*}{$\frac{\alpha}{1}$} & $\mathrm{P}$ & $*$ & $*$ & $*$ & & & $*$ & $*$ & $*$ & $*$ & $*$ & $*$ & $*$ & $*$ & $*$ & & & $*$ & $*$ & $*$ & & & $*$ \\
\hline & $\mathrm{N}$ & $*$ & $*$ & * & $*$ & $*$ & $*$ & $*$ & * & $*$ & * & * & $*$ & $*$ & * & $*$ & $*$ & * & $*$ & * & $*$ & * & * \\
\hline
\end{tabular}

* indica interacción o efectos independientes $(\mathrm{p}<0,05)$ de la condición de protección y del nivel de nutrición.

En ambas etapas de cultivo, el factor nutrición tuvo un efecto de mayor magnitud sobre la ER que la condición de protección (Figura 8). Al considerar la condición de protección, la ER fue mayor cuando el cultivo estuvo protegido, lo cual fue consecuente con una mayor absorción y menor lixiviación de nutrientes. Por otra parte, según la condición de nutrición, aun cuando el aumento del nivel de nutrición incrementó la absorción, la ER se redujo apreciablemente, consecuente con una mayor cantidad de nutriente lixiviado (Figura 8).
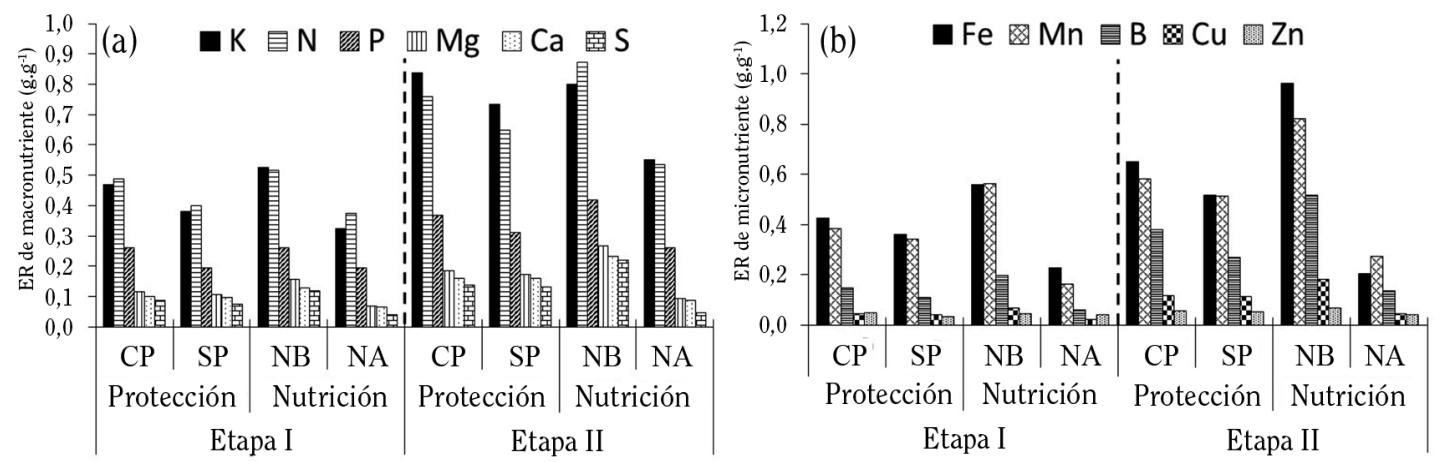

Figura 8. Eficiencia de recuperación de nutrientes (ER; durante la etapa I en época seca y etapa II en época lluviosa de un ciclo de cultivo de culantro coyote hidropónico bajo protección $(\mathrm{CP})$, sin protección (SP), con nutrición baja $(\mathrm{NB})$ y nutrición alta (NA). Alajuela, Costa Rica. 2016.

Durante el experimento, la ER de nutrientes fue mayor en etapa II de invierno que en etapa I de verano. En etapa I, el orden decreciente de la ER de macronutrientes en el cultivo protegido fue $\mathrm{N}: 49 \%>\mathrm{K}: 47 \%>\mathrm{P}: 26 \%>\mathrm{Mg}: 12 \%>\mathrm{Ca}: 10 \%$ $>\mathrm{S}: 9 \%$, mientras que para micronutrientes fue
$\mathrm{Mn}: 60 \%>\mathrm{Fe}: 57 \%>\mathrm{B}: 23 \%>\mathrm{Cu}: 7 \%>\mathrm{Zn}: 5 \%$. Cuando el nivel de nutrición del cultivo fue bajo, el orden decreciente en la ER de macronutrientes fue $\mathrm{N}: 53 \%>\mathrm{K}: 52 \%>\mathrm{P}: 26 \%>\mathrm{Mg}: 16 \%>$ Ca: $13 \%>\mathrm{S}: 12 \%$, mientras que micronutrientes fue Fe:56\%, Mn:56\%, B:20\%, Cu:7\% y Zn: $4 \%$. 
En etapa II, durante época de invierno, en el cultivo protegido la ER de elementos mayores, en orden decreciente fue $\mathrm{K}: 84 \%>$ $\mathrm{N}: 76 \%>\mathrm{P}: 37 \%>\mathrm{Mg}: 19 \%>\mathrm{Ca}: 16 \%>\mathrm{S}: 14 \%$; mientras que para micronutrientes el orden fue $\mathrm{Fe}: 65 \%>\mathrm{Mn}: 58 \%>\mathrm{B}: 38 \%>\mathrm{Cu}: 12 \%>$ $\mathrm{Zn}: 6 \%$. Al considerar el nivel de nutrición, cuando este fue bajo, el orden decreciente en la ER de macronutrientes fue K:87\% $>\mathrm{N}: 85 \%$ $>$ P: $42 \%>$ Mg: $: 27 \%>$ Ca: $23 \%>$ S:22\%; y para micronutrientes el orden fue Fe:96\% $>$ Mn:82\% $>\mathrm{B}: 52 \%>\mathrm{Cu}: 18 \%>\mathrm{Zn}: 7 \%$.

\section{DISCUSIÓN}

En este estudio, la trazabilidad de la cantidad de nutriente absorbido $\left(\mathrm{mg} \cdot \mathrm{m}^{-2}\right)$ y de la $\operatorname{ET}_{c}\left(\mathrm{~L}_{\mathrm{m}} \mathrm{m}^{-2}\right)$, en el cultivo de culantro coyote hidropónico, permitieron determinar la CA de nutrientes (mg.L $\left.\mathrm{L}^{-1}\right)$, un parámetro muy poco investigado aunque novedoso y esencial para optimizar los aportes de agua y nutrientes, según las características morfológicas y fisiológicas del cultivo como consecuencia de la interacción genotipo-clima-manejo agronómico. Sin embargo, los estudios son escasos, únicamente se encontró un estudio general (Sonneveld 2002) y otro en cultivo hidropónico de tomate (Gallardo et al. 2009).

En ambas etapas de cultivo en épocas seca y lluviosa, la absorción de nutrientes (total, TA y CA) fue consecuente con al patrón de crecimiento del cultivo, coincidiendo con valores máximos de MS, IAF y TCC en el periodo de 30 a 40 días. Dicho comportamiento ha sido descrito para cultivos de hoja, donde generalmente las etapas de máxima absorción ocurren en respuesta a los cambios en el patrón de crecimiento según condiciones de clima, etapa fenológica y el manejo agronómico del cultivo (Silber y Bar-Tal 2008). Independiente de la condición de protección y nutrición, el orden de absorción predominante obtenido en este estudio, $\mathrm{K}>\mathrm{N}>\mathrm{Ca}>\mathrm{P}>\mathrm{Mg}$ $>\mathrm{S}>\mathrm{Fe}>\mathrm{Mn}>\mathrm{B}>\mathrm{Zn}>\mathrm{Cu}$, fue consecuente con el orden de absorción obtenido por Bertsch (2003) en cultivo extensivo en suelo. Sin embargo, al comparar dicha absorción según el rendimiento (rollos $\mathrm{m}^{-2}$ ), en general las cantidades absorbidas en el cultivo de culantro hidropónico protegido fueron superiores al cultivo en suelo, excepto para la condición de bajo nivel de nutriente. Sin embargo, estas discrepancias son difíciles de explicar, debido a las diferencias inherentes al sistema de cultivo, al clima y al desconocimiento en el manejo agronómico otorgado al cultivo.

Durante ambas etapas del experimento, la evolución de la $\mathrm{ET}_{\mathrm{c}}$ fue congruente con el patrón de crecimiento y de absorción de nutrientes del cultivo, alcanzando valores máximos entre los 30 y 40 días. Una mayor CA en el cultivo protegido, respecto al de la intemperie, estuvo relacionada con una reducción del exceso de radiación solar, que favoreció un mayor crecimiento (MS, TCC) y un consecuente incremento en la absorción de nutrientes. A la vez, al reducir la cantidad de radiación con la protección de seguido disminuyó la $\mathrm{ET}_{\mathrm{c}}$. Por el contrario, en el cultivo sin protección una mayor radiación solar y de lluvia redujo el crecimiento (MS, TCC) y, secuentemente, disminuyó la absorción de nutrientes; a la vez una mayor radiación solar a la intemperie aumentó la $\mathrm{ET}_{\mathrm{c}}$, lo cual redujo la CA de nutrientes.

Mayor CA en el cultivo bajo protección probablemente fue debido a que la protección redujo los efectos perjudiciales de climas extremos sobre el cultivo. En el cultivo a la intemperie, en etapa I en época seca, el exceso de radiación solar probablemente favoreció la fotooxidación y foto-inhibición del cultivo, mientras que en época de invierno el exceso de lluvia promovió el lavado de nutrientes, lo cual limitó la absorción de nutrientes, que disminuyeron así la CA. Al respecto, Callejas et al. (2016) y Sosa (2006) reportaron que el uso de un $40 \%$ de sombra en culantro coyote incrementó la producción de biomasa y el IAF. Otros estudios demostraron que la $\mathrm{ET}_{\mathrm{c}}$ disminuyó conforme se redujo la radiación solar bajo invernadero en los cultivos de chile dulce (Fernández et al. 2005), tomate y pepino (Lorenzo et al. 2006). 
Por otra parte, en ambas etapas del cultivo, una mayor CA cuando el nivel de nutrientes fue alto, estuvo relacionada con un sustancial incremento en la absorción de nutrientes, debido a mayor producción de MS y TCC, mientras que la $\mathrm{ET}_{\mathrm{c}}$ fue similar entre ambos niveles de nutrición. Estudios realizados en el cultivo de lechuga, un cultivo, en el que similar al culantro el producto cosechable es la hoja, demostraron que conforme aumentó el aporte de nutrientes incrementó la producción de biomasa (Hoque et al. 2010), no obstante, no se indica si mayor biomasa implicó una mejoría en la ER de nutrientes. Algunos estudios reportan valores de ER en cultivos en suelo tales como cereales (Dobermann y Cassman 2002), trigo (García 2004) y maíz (Remache et al. 2017) en rangos que variaron entre 30 y $50 \%$ para el N, 19 a $28 \%$ en $\mathrm{P}$ y de $8,7 \%$ en K. Sin embargo, en cultivos hidropónicos, y específicamente para culantro Coyote, no se encontraron estudios que permitan contrastar los resultados obtenidos. La mayor ER sin afectar el rendimiento, se obtuvo en el cultivo con protección donde el N (ER:49\%) y el P (ER:26\%) estuvieron dentro de los rangos anteriormente reportados, mientras que el $\mathrm{K}$ fue superior (ER:47\%).

De acuerdo con Allen et al. (2006), al incrementar la producción de biomasa, incrementa el consumo hídrico y la absorción de nutrientes. Sin embargo, en el presente estudio se evidenció que la proporcionalidad entre dichos parámetros varió según el nivel de nutrición y de las condiciones climáticas relacionadas con la protección del cultivo. En ambas etapas, el comportamiento mostrado en la TCC, la TA y la $\mathrm{ET}_{\mathrm{c}}$ fue consecuente con la evolución en la CA. Dichos parámetros fueron superiores cuando el cultivo estuvo protegido y el nivel de nutrición fue alto, con un efecto de mayor magnitud debido al nivel de nutrición. Lo anterior, sugiere que, al conocer el patrón de crecimiento del cultivo, junto a la integración del patrón de consumo de agua y de absorción de nutrientes en un parámetro único (CA), contribuiría a ajustar la nutrición en la producción de culantro coyote en sistema hidropónico en las condiciones específicas de cada región y, de esta forma, mejorar la ER de nutrientes.

En cultivos hidropónicos, estudios realizados por Silber y Bar-Tal (2008) sugieren que el flujo de masas es el mecanismo de absorción primario de agua y nutrientes en hidroponía, lo cual reafirma la importancia de las curvas de CA en cultivos hidropónicos. Coherente con el manejo de riegos frecuentes y de corta duración realizado en el presente estudio, los autores demostraron que dicho manejo elimina la zona de agotamiento en el bulbo húmedo, debido a la reposición frecuente de solución nutritiva en la zona adyacente a la superficie de las raíces, y disminuye el gradiente de concentración de nutrientes entre la solución del medio y la interfaz de las raíces. Como resultado, esto incrementa el transporte y la absorción de nutrientes por medio del flujo de masas y disminuye el papel de la difusión e interceptación como mecanismos de absorción de nutrientes.

De acuerdo con el planteamiento anterior, el incremento en la absorción de nutrientes, en el presente cultivo hidropónico de culantro coyote bajo protección, probablemente se debió a que el flujo de transpiración, como mecanismo prioritario de absorción de nutrientes (Silber y Bar-Tal 2008), fue el principal componente de la $\mathrm{ET}_{\mathrm{c}}$, mientras que la evaporación desde la superficie húmeda y expuesta del sustrato fue menos relevante. Por el contrario, en los cultivos sin protección, la evaporación incrementó y pasó a ser el principal componente de la $\mathrm{ET}_{\mathrm{c}}$, principalmente en las primeras semanas iniciales del cultivo, cuando la cobertura foliar aún es baja. Además, en los cultivos protegidos, hubo mayor producción de MS e IAF, lo cual está directamente relacionado con una mayor transpiración (Qiu et al. 2013, Di Benedetto y Tognetti 2016). El hecho de que el flujo de masas sea el principal mecanismo de absorción de nutrientes en hidroponía, y que está principalmente regulado por las condiciones climáticas, resalta una vez más la importancia de la determinación de las curvas de CA en cultivos 
hidropónicos, en función de las condiciones climáticas y las prácticas de manejo.

En ambas etapas del experimento, tanto en época de verano como en invierno, la protección del cultivo favoreció un mayor crecimiento expresado en un incremento de IAF, MS y TCC. Como efecto, incrementó la absorción, disminuyó la lixiviación y mejoró la ER de nutrientes. Además, cuando el nivel de nutrición fue alto, aunque incrementó la absorción, simultáneamente aumentó apreciablemente la lixiviación, lo que derivó la reducción de la ER.

Una mayor ER al reducir el nivel de nutrición, se obtuvo a expensas de una disminución en la producción de MS (rendimiento); mientras que cuando se aumentó el nivel de nutrición incrementó el rendimiento a expensas de una drástica disminución en la ER. Al respecto, Roberts (2008) sugiere que existe una eficiencia óptima económica, en la cual se garantiza la eficiencia nutricional, la económica (rendimiento) y la ambiental, asimismo se asegura la sostenibilidad de los sistemas de producción.

\section{CONCLUSIONES}

En los cultivos bajo protección con sarán en verano y con plástico en invierno, incrementó el crecimiento en términos de materia seca, índice de área foliar y tasa de crecimiento del cultivo, lo cual como consecuencia aumentó la absorción, disminuyó la lixiviación de nutrientes y mejoró así la eficiencia de recuperación. Al mismo tiempo que aumentó la absorción de nutrientes, disminuyó la evapotranspiración del cultivo, y como efecto, incrementó la concentración de absorción de nutrientes. Por otra parte, en los cultivos que recibieron un nivel de nutrición alto, a pesar de que incrementó la absorción, paralelamente hubo mayor lixiviación y, por tanto, se redujo la eficiencia de recuperación. Simultáneamente, hubo mayor concentración de absorción de nutrientes, debido a un sustancial incremento en la absorción, ya que la evapotranspiración del cultivo fue similar en ambos niveles de nutrición.
De acuerdo con lo descrito anteriormente, el cultivo hidropónico de culantro coyote bajo protección, y con alto nivel de nutrición, representa una excelente alternativa ante la producción convencional de cultivo en suelo a la intemperie, ya que ambos favorecen un incremento en la producción de biomasa que se traduce en mayor rendimiento. Además, la protección favoreció una mayor eficiencia de recuperación debido a un mayor crecimiento y absorción de nutrientes, y a la reducción de la lixiviación de nutrientes. Sin embargo, la aplicación de alta concentración de nutrientes en solución nutritiva, una práctica habitual en producción hidropónica en bancales a la intemperie, es el factor con el efecto de mayor magnitud en el aumento de la lixiviación de donde proviene la disminución en la eficiencia de recuperación de nutrientes. Según lo anterior, las curvas de absorción en términos de concentración de absorción de nutrientes, determinadas en el presente estudio, representan una herramienta con gran potencial para optimizar las concentraciones de nutrientes en el agua aplicada (mg.L $\left.\mathrm{L}^{-1}\right)$, según la demanda nutricional e hídrica en función del crecimiento y las condiciones climáticas del cultivo de culantro coyote protegido.

En este estudio, el patrón de crecimiento y las características morfo-fisiológicas del cultivo en respuesta a la condición de protección y de nutrición, determinó la curva de concentración de absorción (mg. $\mathrm{L}^{-1}$ ) en términos mg de nutrientes por litro de agua requerido por el cultivo hidropónico de culantro coyote. La interacción entre el patrón de crecimiento del cultivo y la respectiva absorción de nutrientes y agua, integrados en la concentración de absorción de nutrientes, varío con las condiciones específicas del tipo de protección, según época y con el manejo de la nutrición.

\section{LITERATURA CITADA}

Allen, RG; Pereira, LS; Raes, D; Smith, M. 2006. Evapotranspiración del cultivo. Guías para la determinación de los requerimientos de agua de los cultivos. Estudio FAO-56. Riego y Drenaje. Roma, Italia. $323 \mathrm{p}$. 
Ansorena, MJ. 1994. Sustratos. Propiedades y caracterización. Madrid, España, Mundi Prensa. $167 \mathrm{p}$.

Bertsch, F. 2003. Absorción de nutrimentos por los cultivos. San José, Costa Rica, Asociación Costarricense de la Ciencia del Suelo. 308 p.

Callejas, I; Cerritos, R; Rauda, M. 2016. Caracterización morfoagronómica e invertebrados asociados al acapate (Apaiaceae: Eryngium foetidum L.). Facultad de Ciencias Agronómicas. Universidad de El Salvador, El Salvador. 97 p.

Caseira-Posada, F. 2007. Foto inhibición: Respuesta fisiológica de los vegetales al estrés por exceso de luz. Una revisión. Photoinhibition: Physiological response of plants to high irradiance stress. A review. Revista Colombiana de Ciencias Hortícolas 1(1):114 123.

Cerdas, M; Montero, M. 2016. Efecto de cambios en manejo poscosecha sobre la calidad y vida útil del culantro coyote para exportación. Agronomía Costarricense 40(1):51-64.

Di Benedetto, A; Tognetti, J. 2016. Técnicas de análisis de crecimiento de plantas: su aplicación a cultivos intensivos. Ria 42(1900):258-282.

Di Rienzo, JA; Macciavelli, RE; Casanoves. F. 2012. Modelos Lineales Mixtos: aplicaciones en InfoStat. Córdoba, Argentina ,Universidad de Córdoba. 193 p.

Dobermann, A; Cassman, K. 2002. Plant nutrient management for enhanced productivity in intensive grain production systems of the United States and Asia (en línea). Plant and Soil. 247(1):153-175. Consultado ago. 2020. Disponible en https://www. jstor.org/stable/24123904?seq=1

Doberman, AR. 2005. Nitrogen Use Efficiency State of the Art (en línea). University of Nebraska Lincoln, Agronomy Horticulture. Nebraska, USA. 316:16. Consultado feb. 2020. Disponible en http:// digitalcommons.unl.edu/agronomyfacpub/316

Fernández, MD; Gallardo, M; Bonachela, S; Orgaz, F; Thompson, RB; Fereres, E. 2005. Water use and production of a greenhouse pepper crop under optimum and limited water supply. J. Hortic. Sci. Biotech. 80(1):87-96.

Gallardo, M; Thompson, RB; Rodríguez, JS; Rodríguez, F; Fernández, MD; Sánchez, JA; Magán, JJ. 2009. Simulation of transpiration, drainage, $\mathrm{N}$ uptake, nitrate leaching, and $\mathrm{N}$ uptake concentration in tomato grown in open substrate. Agric. Water Manag. 96(12):1773-1784.

García, F. 2004. Advances in nutrition management of wheat. Proceedings Wheat National Symposium, Mar del Plata, 13-14 May 2004. Federation of Grain Traders of Argentina. p. 2-10.

Hoque, M; Ajwa, H; Othman, M; Smith, R; Cahn, M. 2010. Yield and postharvest quality of lettuce in response to nitrogen, phosphorus, and potassium fertilizers. Hort. Sci. 45(10):1539-1544.

Jara, D. 2016. Evaluación de tres híbridos de pimiento (Capsicum annum L.) cultivados en hidroponía con tres mezclas de sustrato. Tesis Ing. Agr. Universidad de Guayaquil. Guayaquil, Ecuador. 46 p.

Krug, H. 1999. Environmental influences development, growth and yield. In Physiology of Vegetable Crops. Edited by $\mathrm{H}$ Wien, Cornell University, USA. p. 101-126.

Lavagni, G; Rodríguez, G. 2009. El Culantro Coyote, producto de exportación. Alimentaria 102:30-36.

Lorenzo, P; García, ML; Sánchez-Guerrero, MC; Medrano, E; Caparrós, I; Giménez, M. 2006. Influence of Mobile Shading on Yield, Crop Transpiration and Water Use Efficiency. Acta Horticulturae 719:471 478 .

Murray, MS. 2008. Using degree-days to time treatments for insect pests. Fact Sheet. Utah State University Extension, Utah Plant Pest Diagnostic Laboratory. Utah, United States. IPM 05-08. 5 p.

Qiu, R; Song, J; Du, T; Kang, S; Tong, L; Chen, R; Wu, L. 2013. Response of evapotranspiration and yield to planting density of solar greenhouse grown tomato in northwest China. Agric. Water Manag. 130:44-51.

Remache, M; Carrillo, M; Mora, R; Durango, W; Morales, F. 2017. Absorción de macronutrientes y eficiencia del N, en híbrido promisorio de maíz. Agronomía Costarricense 41(2):103-115.

Resh, H. 2001. Cultivos hidropónicos, nuevas técnicas de producción. $5^{\text {a }}$ edición. Madrid, España, MundiPrensa. 547 p.

Roberts, TL. 2008. Improving Nutrient Use Efficiency. Turk J Agric For. 32:177-182.

Rodríguez, DA; Hoyos, M; Chang, M. 2001. Soluciones nutritivas en hidroponía. Formulación y preparación. $2^{\mathrm{a}}$ edición. Lima, PE, Universidad Agraria La Malina, Centro de Investigación de Hidroponía e Investigación Mineral. 97 p.

Rojas, J; Paniagua, F. 2015. Comportamiento agronómico de Capsicum annuum L., Lycopersicon esculentum M. y Cucumis melo L. bajo cultivo protegido hidropónico utilizando la solución universal de Steiner. Tesis Lic. San Carlos, Costa Rica, Tecnológico de Costa Rica. 98 p.

Silber, A; Bar-Tal, A. 2008. Nutrition of Substrate-grown Plants. In Raviv, M; Lieth, JH (eds.). Soilless Culture: Theory and Practice. San Diego: Academic Press, p. 291-340.

Sonneveld, C. 2002. Composition of nutrient solutions. In Savvas, D; Passam, H (eds.). Hydroponic Production of Vegetables and Ornamentals. Athens, Greece, Embryo Publications. p.178-210.

Sosa, E. 2006. Evaluación de la producción de culantro coyote (Eryngium foetidum Lineo) en tres ambientes diferentes y dos tipos de fertilización en la zona 
atlántica de Costa Rica. Tesis Lic. Guácimo, Costa Rica, Universidad EARTH. 55 p.

Soto, F; Ramírez, A. 2005. Hidroponía. Centro Nacional Especializado Granja Modelo. San José, Costa Rica, Instituto Nacional de Aprendizaje. 109 p.

Soto, F; Thompson, RB; Granados, MR; Martínez-Gaitán, C; Gallardo, M. 2018. Simulation of agronomic and nitrate pollution related parameters in vegetable cropping sequences in Mediterranean greenhouses using the EU-Rotate_N model. Agric. Water Manag. 199:175-189.
UNE-EN-13041 (Una Norma Española - European Norm). 2007. Mejoradores de suelo y sustratos de cultivo. Determinación de las propiedades físicas: densidad aparente seca, volumen de aire, volumen de agua, valor de contracción y porosidad total. Madrid, España, Asociación Española de Normalización y Certificación (AENOR). 24 p.

Urrestarazu, GM. 2015. Manual práctico del cultivo sin suelo e hidroponía. Almería, España, MundiPrensa. 278 p. 